\title{
Supersymmetric Models with Higher Dimensional Operators
}

\author{
I. Antoniadis ${ }^{a, b}$, E. Dudas ${ }^{b, c}$, D. M. Ghilencea ${ }^{d}$ \\ ${ }^{a}$ Department of Physics, CERN - Theory Division, 1211 Geneva 23, Switzerland. \\ ${ }^{b}$ CPHT, UMR du CNRS 7644, École Politechnique, 91128 Palaiseau Cedex, France. \\ ${ }^{c} L P T$, UMR du CNRS 8627, Bât 210, Université de Paris-Sud, 91405 Orsay Cedex, France \\ ${ }^{d}$ Rudolf Peierls Centre for Theoretical Physics, University of Oxford, \\ 1 Keble Road, Oxford OX1 3NP, United Kingdom.
}

\begin{abstract}
In $4 \mathrm{D}$ renormalisable theories, integrating out massive states generates in the low energy effective action higher dimensional operators (derivative or otherwise). Using a superfield language it is shown that a $4 \mathrm{D} \mathrm{N}=1$ supersymmetric theory with higher derivative operators in either the Kahler or the superpotential part of the Lagrangian and with an otherwise arbitrary superpotential, is equivalent to a $4 \mathrm{D} \mathrm{N}=1$ theory of second order (i.e. without higher derivatives) with additional superfields and renormalised interactions. We provide examples where a free theory with trivial supersymmetry breaking provided by a linear superpotential becomes, in the presence of higher derivatives terms and in the second order version, a non-trivial interactive one with spontaneous supersymmetry breaking. The couplings of the equivalent theory acquire a threshold correction through their dependence on the scale of the higher dimensional operator(s). The scalar potential in the second order theory is not necessarily positive definite, and one can in principle have a vanishing potential with broken supersymmetry. We provide an application to MSSM and argue that at tree-level and for a mass scale associated to a higher derivative term in the $\mathrm{TeV}$ range, the Higgs mass can be lifted above the current experimental limits.
\end{abstract}




\section{Introduction}

The search for Physics beyond the Standard Model (SM) within the framework of effective field theories addresses in particular the role of higher dimensional operators and their possible experimental footprints. In effective field theories and in models compactified to $4 \mathrm{D}$ these operators are a common presence. A special class of operators is that of higher derivative operators, whose role has not been investigated in great detail. In this work both classes of higher dimensional operators (derivative or otherwise) are included. One motivation for considering the study of such operators is that they can be generated at low energy (below some scale) by renormalisable new physics at this scale, after integrating out massive degrees of freedom. Such operators are also generated dynamically by radiative corrections even in the simplest orbifold compactifications, see for example [1, 2, 3, 4, 5]. Such operators can be generated by either bulk or brane-localised interactions, when integrating out the loop corrections of modes associated with the compactification. For example they are generated by one-loop gauge interactions in $6 \mathrm{D}$ compactifications [1, 3, 4, 5] and by one-loop localised superpotential interactions in 5D or 6D orbifolds [2]. These operators respect all the symmetries of the models considered and their generation as counterterms by quantum effects prompted, in part, the present analysis.

In the context of effective field theories higher dimensional derivative operators were investigated in the past; they were studied in the framework of Randall-Sundrum models [6], have implications for cosmology [7, 8, 9], phase transitions and Higgs models [10, 11, 12, 13, supergravity/higher derivative gravity [14]-[24], string theory [25, 26], cosmological constant [27], implications for the UV regime [28], for instabilities [29], and model building [30]-33]. Applications of theories with such operators also included their role in regularisation methods debated in [34, 35, 36, 37]. Interacting theories with higher derivatives involve the presence of ghosts which can bring in difficult issues (for example unitarity violation), some of which were studied in [38, 39]. Such issues are actually common, since the presence of ghosts is also familiar in standard Pauli-Villars regularisation method of 4D theories (see [40] and references therein). Provided that the scale of the higher derivative operators is high-enough the associated effects are suppressed at low energies. In theories with higher derivative operators the vacuum-to-vacuum amplitude is well-defined (no exponential growth) provided that the ghost fields are not asymptotic states [41] (i.e. are present as loop states only). In this case the vacuum-to-vacuum amplitude (and therefore Green functions) tends to that of second order 
theory in the decoupling limit of a very high scale of these operators. One looses unitarity near this high scale, but can never produce a negative-norm state or negative probability [41].

The purpose of this work is to study the case of generic 4D N=1 supersymmetric models with higher derivative terms in either its Kahler part or in the superpotential, for an otherwise arbitrary superpotential and field content. Using a supersymmetric formulation it is shown that such models are in fact equivalent to a second order theory with renormalised interactions and additional (ghost) superfields manifestly present in the action. The couplings of the new, second order theory, acquire dynamically, at the tree level, a dependence on the scale of the higher dimensional operators, via wavefunction rescaling. For specific assumptions for the analytical continuation of the theory from Minkowski to Euclidean metric and in the absence of additional higher dimensional non-derivative operators, we argue that the new theory can be renormalisable for the case of higher derivative terms considered.

Supersymmetry breaking is also considered and in this higher derivative operators can play an interesting role. It is showed that apparently un-interesting models with higher derivative terms, without interactions and with trivial supersymmetry breaking in the decoupling limit of the higher derivative terms, are in fact interacting in the new, second order formulation, and also have spontaneous supersymmetry breaking à la O'Raifeartaigh [42].

Independent of the exact nature of the ghost (super)fields mentioned earlier (loop only or asymptotic states) the method we develop enables us to estimate perturbatively the effects of high-scale physics due to higher derivative operators on low energy observables. The presence of ghost superfields leads to a scalar potential which is not necessarily positive definite and one could in principle have a positive, negative or even vanishing scalar potential for broken supersymmetry. The last case can be relevant for the cosmological constant problem [43].

We would like to emphasize one point, briefly mentioned in the first paragraph, which we consider extremely important regarding the origin of the higher dimensional operators (derivative or otherwise). Consider a $4 \mathrm{D}$ renormalisable theory with a massive superfield $\chi$, of mass $M_{*} \gg m$, with

$$
\mathcal{L}_{1}=\int d^{4} \theta\left[\Phi^{\dagger} \Phi+\chi^{\dagger} \chi\right]+\left\{\int d^{2} \theta\left[\frac{M_{*}}{2} \chi^{2}+m \Phi \chi+\mathcal{W}(\Phi)\right]+\text { h.c. }\right\}
$$

After solving the eq of motion for $\chi$, one immediately finds

$$
\chi=\frac{1}{M_{*}}\left[-m \Phi-\frac{m}{4 M_{*}} \bar{D}^{2} \Phi^{\dagger}+\frac{1}{16} \frac{-m}{M_{*}^{2}} \bar{D}^{2} D^{2} \Phi-\frac{m}{64 M_{*}^{3}} \bar{D}^{2} D^{2} \bar{D}^{2} \Phi^{\dagger}+\cdots\right]
$$


which if plugged back into the Lagrangian brings about terms of the form

$$
\mathcal{L}_{1} \supset \int d^{4} \theta\left\{\frac{m^{2}}{8 M_{*}^{3}}\left[\Phi D^{2} \Phi+\text { h.c. }\right]+\frac{m^{2}}{16 M_{*}^{4}}\left(\bar{D}^{2} \Phi^{\dagger}\right)\left(D^{2} \Phi\right)+\cdots\right\}
$$

Therefore, integrating out massive (super)fields generated higher derivative operators in the low energy effective action valid below the scale $M_{*}$. Since the original theory was free of ghosts, the same remains true about the low energy action as long as one considers the whole series of higher dimensional operators in (3). However, in an effective theory study and for practical purposes, one is often restricting the analysis to a finite set of higher dimensional operators, of lowest order in $1 / M_{*}$. The consequence of this is the presence of ghosts in the action, as a sign that the theory is incomplete in the UV (i.e. physics above $M_{*}$ ). From an effective field theory point of view, as we adopt in this paper, the absence of a UV completion is assumed anyway, therefore we are not addressing, in our discussion below, the more conceptual problems that ghosts can eventually bring. Our goal is to show however, how one can investigate effective theories with higher derivative operators in difficult cases such as when the original, high energy action is unknown. For a discussion of related issues see [44].

The plan of the paper is as follows. Section 2 introduces the lowest-order higher derivative terms in superfield language. In Section 3 we consider a $4 \mathrm{D} \mathrm{N}=1$ model with higher derivative kinetic term and show its equivalence to a second order theory. The analysis is done firstly for a specific superpotential, to set out our method of "unfolding" the fourth order theory into an equivalent, second order one. This is then generalised to an arbitrary superpotential (without derivative terms). In Section 4 we discuss the case of higher derivative terms in the superpotential which is otherwise arbitrary, and perform a similar analysis, to find the equivalent second order theory. The case of spontaneous supersymmetry breaking and the form of soft terms is briefly discussed in Section [5. In Section 6 we discuss briefly an application to MSSM with higher derivative operators and show that the lightest Higgs mass acquires corrections of order $2 \mu / M_{*}$, which are sizable for mass scales $M_{*}$ suppressing the operator in the $10 \mathrm{TeV}$ range and that can raise the Higgs mass above the current experimental bounds. We end with the conclusions and a short Appendix.

\section{Higher dimensional operators : general considerations}

The framework of our analysis is that of $4 \mathrm{D} \mathrm{N}=1$ supersymmetric models. In such models, one can commonly have higher dimensional operators, which can involve higher derivatives 
or not. Let us consider the last case. Despite rendering a model non-renormalisable, such operators are important for phenomenology. Our analysis in this paper is valid in the presence of such operators in the superpotential and does not make explicit reference to them. If such operators are present in the Kahler term, they again do not affect the analysis below. This is because our analysis will only involve transformations (change of "basis") of fields having higher (super)derivatives and of their derivatives, to a new basis of superfields where such derivatives are absent. Since we will provide the relations between the two bases of fields, old and new, the higher dimensional operators referred to earlier, if they involve a field which 1 underwent such a transformation, will immediately be known in the new, equivalent basis. This will become clearer in the next sections. Briefly, higher dimensional operators are spectators under the transformations we consider, allowing our analysis to be general.

Let us now consider, in the $4 \mathrm{D} \mathrm{N}=1$ supersymmetric context, the possible operators involving (super)derivatives, to the lowest orders. Such operators are less studied in the literature. They can be constructed using combinations of powers of $D^{2}, \bar{D}^{2}$ and of the superfields denoted $\Phi_{j}$. For the lowest powers of $D, \bar{D}$, these are (integrated appropriately over Grassmann space):

$$
\begin{aligned}
& \text { (a) } \int d^{2} \theta \Phi_{i}\left(\bar{D}^{2} \Phi_{j}^{\dagger}\right)+h . c ., \quad(b) \int d^{4} \theta\left[\Phi_{i}\left(D^{2} \Phi_{j}\right)+h . c .\right] \sim \int d^{2} \theta \Phi_{i} \square \Phi_{j}+h . c \\
& \text { (c) } \int d^{4} \theta \Phi_{i}^{\dagger} \bar{D}^{2} D^{2} \Phi_{j} \sim \int d^{4} \theta \Phi_{i}^{\dagger} \square \Phi_{j}, \quad(d) \int d^{4} \theta \Phi_{i}^{\dagger} \Phi_{j} D^{2} \Phi_{k}
\end{aligned}
$$

(a) is just $\int d^{4} \theta\left(\Phi_{i}^{\dagger} \Phi_{j}+\right.$ h.c.), (b) has dimension 5, (c) and (d) have dimension 6; (b) is studied in section 4, (c) is studied in section 3 while (d) can be treated in a similar way, see section 5 . Further, one could also consider

$$
\text { (e) } \int d^{2} \theta \Phi^{n}\left(\bar{D}^{2} \Phi_{j}^{\dagger}\right)^{p}+\text { h.c. }
$$

of dimension $2 p+n+1$. This generalises (a) and can also be treated following the same method as in Section 4 and for $n+p \leq 3$ can actually be renormalisable. In fact (b), (c), (d), can also be renormalisable (see Section 5.4) despite having dimension 5, 6, 6 respectively. The renormalisability will be seen once we write a theory with such operators as an equivalent, second order theory involving only dimension 4 operators. The renormalisable character of the new, equivalent theory, adds support to their study and partly motivated their analysis.

\footnotetext{
${ }^{1}$ having a (super)derivative
} 


\section{$3 \quad$ Effects of higher derivative kinetic terms.}

\subsection{The Wess-Zumino model with higher derivative terms.}

We start with the Wess-Zumino Lagrangian with a higher derivative term, with $\Phi \equiv(\phi, \psi, F)$ :

$$
\begin{aligned}
\mathcal{L} & =\int d^{4} \theta \Phi^{\dagger}(1+\xi \square) \Phi+\left\{\int d^{2} \theta\left[\frac{1}{2} m \Phi^{2}+\frac{1}{3} \lambda \Phi^{3}\right]+c . c .\right\} \\
& =F^{*}(1+\xi \square) F-\phi^{*} \square(1+\xi \square) \phi+i \partial_{\mu} \bar{\psi} \bar{\sigma}^{\mu}(1+\xi \square) \psi \\
& +\left(\frac{1}{2} m(2 \phi F-\psi \psi)+\lambda\left(\phi^{2} F-\phi \psi^{2}\right)+\text { c.c. }\right)
\end{aligned}
$$

where $\xi \equiv 1 / M_{*}^{2}$, with $M_{*}$ the scale where the higher dimensional operator becomes relevant. We assume that $M_{*}^{2}$ is significantly larger than all other scales in the theory (like $m^{2}$ or vev's of the fields). Due to the presence of the term proportional to $\xi$, the auxiliary field $F$ is now dynamica 2 . The spectrum of scalar states is found from the poles of the propagators $\left\langle\phi \phi^{*}\right\rangle$, $\left\langle F F^{*}\right\rangle$. From these, one finds the masses as the roots of $\square(1+\xi \square)^{2}+m^{2}=0$, given by

$$
\begin{aligned}
m_{1}^{2} & =m^{2}\left[1+2 \xi m^{2}+7\left(\xi m^{2}\right)^{2}+\mathcal{O}\left(\left(\xi m^{2}\right)^{5 / 2}\right)\right] \\
m_{2,3}^{2} & =m^{2}\left[\frac{1}{\xi m^{2}} \pm \frac{1}{\sqrt{\xi m^{2}}}-\frac{1}{2} \pm \frac{5 \sqrt{\xi m^{2}}}{8}-\xi m^{2}+\mathcal{O}\left(\left(\xi m^{2}\right)^{3 / 2}\right)\right]
\end{aligned}
$$

The last two masses correspond to two ghost states associated with $F$ and $\square \phi$, see their negative kinetic terms. These values will be needed in the next section. For later reference, the scalar potential in the limit $\xi=0$ is: $V(\phi)=\left|m \phi+\lambda \phi^{2}\right|^{2}$ which has two supersymmetric ground states $V=0,\langle F\rangle=0$ situated at $\langle\phi\rangle=\left\langle\phi^{*}\right\rangle=0$ and $\langle\phi\rangle=-m / \lambda,\left\langle\phi^{*}\right\rangle=-m / \lambda^{*}$. The mass matrix in the basis $\left(\phi, \phi^{*}\right)$ has eigenvalues $m_{1,2}^{2}=m^{2}$. A saddle point is located at $\phi=-m /(2 \lambda), \phi^{*}=-m /\left(2 \lambda^{*}\right)$, where $m_{1,2}^{2}= \pm m^{2} / 2$. One can use the above Lagrangian for calculations, including loop effects in the presence of the higher derivative operator [28]. However, it would be preferable to have a better understanding of the role of such operator and, if possible, a formulation of such models as a second order theory. This could prove very helpful for applications.

\footnotetext{
${ }^{2}$ By supersymmetry, this will require, in a second order formulation of this theory to be found in the following, the introduction of an additional superfield, see later
} 


\subsection{The higher derivative Wess-Zumino model as a second order theory}

In this section we show in a manifest supersymmetric way that a Wess-Zumino model with a higher derivative term is equivalent to a second order theory with new superfields and renormalised interactions. The results are then generalised to an arbitrary superpotential. One has (her $3^{3} s_{1} \equiv \pm 1$ )

$$
\begin{aligned}
\mathcal{L} & =\int d^{4} \theta \Phi^{\dagger}\left(1+s_{1} \xi \square\right) \Phi+\left\{\int d^{2} \theta\left[\frac{m}{2} \Phi^{2}+\frac{\lambda}{3} \Phi^{3}\right]+\text { h.c. }\right\} \\
& =\int d^{4} \theta\left[\Phi^{\dagger} \Phi-\frac{s_{1} \xi}{16} D^{2} \Phi \bar{D}^{2} \Phi^{\dagger}\right]+\left\{\int d^{2} \theta\left[\frac{m}{2} \Phi^{2}+\frac{\lambda}{3} \Phi^{3}\right]+\text { h.c. }\right\}
\end{aligned}
$$

Introduce

$$
\begin{aligned}
\Phi & =a_{1} \Phi_{1}+a_{2} \Phi_{2} \\
\Phi_{D} \equiv m^{-1} \bar{D}^{2} \Phi^{\dagger} & =b_{1} \Phi_{1}+b_{2} \Phi_{2}
\end{aligned}
$$

where we used that $\Phi_{D} \equiv\left(\phi_{D}, \psi_{D}, F_{D}\right)$ is itself a chiral superfield. The $2 \times 2$ matrix of coefficients $a_{1,2}, b_{1,2}$ must be unitary, to maintain the eigenvalue problem. A useful parametrisation for the unitary matrix is $a_{1}=\cos \theta \exp \left(i h_{1}\right), a_{2}=\sin \theta \exp \left(-i\left(h-h_{1}\right)\right)$, $b_{1}=-\sin \theta \exp \left(i\left(h-h_{1}\right)\right), b_{2}=\cos \theta \exp \left(-i h_{1}\right)$ where $\theta, h, h_{1}$ are real. In principle one could work with a simplified assumption $\left(a_{1}=b_{2}=1, a_{2}=b_{1}=0\right)$ since the difference is a rotation in the superfield space. For generality we keep the matrix entries in the above non-trivial parametrization, to show explicitly that the final results are independent of a such particular choice 4. Eq.(9) gives a constraint

$$
m^{-1}\left[a_{1}^{*} \bar{D}^{2} \Phi_{1}^{\dagger}+a_{2}^{*} \bar{D}^{2} \Phi_{2}^{\dagger}\right]=b_{1} \Phi_{1}+b_{2} \Phi_{2}
$$

To account for this, we must introduce an additional contribution $\Delta L$ to the Lagrangian, where the Lagrange multiplier is a new chiral superfield $\Phi_{3}$ (therefore $\bar{D} \Phi_{3}=0$ ). We then have 5

\footnotetext{
${ }^{3}$ Only $s_{1}=+1$ gives a bounded Euclidean action, but we keep $s_{1}$ only to trace its effects in formulae below.

${ }^{4}$ For an easier, more transparent first reading one could set in the following $a_{1}=b_{2}=1, a_{2}=b_{1}=0$.

${ }^{5}$ Without any restriction of generality, we used the scale $m$ in eq.(9), (10), introduced for dimensional reasons. In principle one can use there any other finite, non-zero mass scale of the theory, $m_{q}$. However, our use of $m$ instead of $m_{q}$ only amounts to a simple re-definition, always allowed (and assumed to have been made), of our original parameter $\xi \rightarrow \xi m_{q}^{2} / m^{2}$, as it can easily be seen from inserting (9) in eq.(8).
} 


$$
\Delta \mathcal{L}=\int d^{2} \theta\left[m^{-1}\left(a_{1}^{*} \bar{D}^{2} \Phi_{1}^{\dagger}+a_{2}^{*} \bar{D}^{2} \Phi_{2}^{\dagger}\right)-\left(b_{1} \Phi_{1}+b_{2} \Phi_{2}\right)\right] \Phi_{3} m_{*}+h . c ., \quad m_{*} \equiv \frac{\sqrt{\xi} m^{2}}{4}
$$

Above $m_{*}$ was introduced for dimensional reasons; since the constraint should be removed in the absence of the higher derivative term $(\xi \rightarrow 0)$ in the original action, $m_{*}$ should be proportional to $\xi$; further, each of the $D^{2} \Phi$ or $\bar{D}^{2} \Phi^{\dagger}$ derivatives comes with a $\sqrt{\xi} / 4$, see eq.(8), and with these observations one then obtains the above expression for $m_{*}$. With $\mathcal{L}^{\prime}=\mathcal{L}+\Delta \mathcal{L}$, then

$$
\begin{aligned}
\mathcal{L}^{\prime} & =\int d^{4} \theta\left[\rho_{1} \Phi_{1}^{\dagger} \Phi_{1}+\left(\rho_{2} \Phi_{1}^{\dagger} \Phi_{2}+\rho_{2}^{*} \Phi_{2}^{\dagger} \Phi_{1}\right)+\left(\rho_{3} \Phi_{1}^{\dagger} \Phi_{3}+\rho_{4} \Phi_{2}^{\dagger} \Phi_{3}+\text { h.c. }\right)+\rho_{5} \Phi_{2}^{\dagger} \Phi_{2}\right] \\
& +\left\{\int d^{2} \theta\left[\frac{m}{2}\left(a_{1} \Phi_{1}+a_{2} \Phi_{2}\right)^{2}+\frac{\lambda}{3}\left(a_{1} \Phi_{1}+a_{2} \Phi_{2}\right)^{3}-m_{*} \Phi_{3}\left(b_{1} \Phi_{1}+b_{2} \Phi_{2}\right)\right]+\text { h.c. }\right\}
\end{aligned}
$$

where

$$
\begin{array}{rlrl}
\rho_{1} & =\left|a_{1}\right|^{2}-\frac{s_{1}}{16}\left|b_{1}\right|^{2} \xi m^{2}, & \rho_{2}=a_{1}^{*} a_{2}-\frac{s_{1}}{16} b_{1}^{*} b_{2} \xi m^{2}, & \\
\rho_{3}=-4 \frac{m_{*}}{m} a_{1}^{*}, & \rho_{4}=-4 \frac{m_{*}}{m} a_{2}^{*}, & \rho_{5}=\left|a_{2}\right|^{2}-\frac{s_{1}}{16}\left|b_{2}\right|^{2} \xi m^{2}(13)
\end{array}
$$

We therefore "traded" the higher derivative term in the original action, for an additional superfield $\Phi_{2}$ plus a constraint, which generated in turn the presence of $\Phi_{3}$. After using the eq of motion (in terms of superfields) for $\Phi_{3} \equiv\left(\phi_{3}, \psi_{3}, F_{3}\right)$ one immediately find 6 : 7 (after using the definition of $\rho_{3,4}$ ) that

$$
m_{*}\left\{m^{-1}\left[a_{1}^{*} \bar{D}^{2} \Phi_{1}^{\dagger}+a_{2}^{*} \bar{D}^{2} \Phi_{2}^{\dagger}\right]-\left[b_{1} \Phi_{1}+b_{2} \Phi_{2}\right]\right\}=0 .
$$

For $m_{*} \neq 0$, this immediately recovers the initial constraint (10), while if $m_{*} \propto \sqrt{\xi} \rightarrow 0$ the constraint $\Delta \mathcal{L}$ of (11) is vanishing, as it should be the case since in this case there is no higher derivative term in (8) .

\footnotetext{
${ }^{6}$ with $-4 \int d^{4} x d^{4} \theta f(x, \theta, \bar{\theta})=\int d^{4} x d^{2} \theta \bar{D}^{2} f(x, \theta, \bar{\theta}), f$ arbitrary.

${ }^{7}$ For later use let us mention that in our conventions

$$
\bar{D}^{2} \Phi^{\dagger} \equiv\left(-4 F^{*} ;-4 i \not \bar{\psi} ; 4 \square \phi^{*}\right), \quad \Phi \equiv(\phi, \psi, F)
$$
}


Also, from eq.(12) one can integrate out $\Phi_{2}, \Phi_{3}$ by using the their equation of motion, to recover the original lagrangian (8). To see this easily one uses their eqs of motion in superfield form and that: $-4 \int d^{4} x d^{4} \theta f(x, \theta, \bar{\theta})=\int d^{4} x d^{2} \theta \bar{D}^{2} f(x, \theta, \bar{\theta})$ and $\bar{D}^{2} D^{2}=-16 \square$.

We diagonalise the hermitian matrix $\mathcal{M}$ of the coefficients of $\mathrm{D}$ terms in (12), in the basis 8 $\left(\Phi_{1}, \Phi_{2}, \Phi_{3}\right)$. Its eigenvalues $\nu_{1,2,3}$ are real, given by the roots of

$$
\nu^{3}+\nu^{2} c_{3}+\nu c_{2}+c_{1}=0
$$

where

$$
\begin{aligned}
c_{3} & =-\frac{1}{16}\left[16\left(\left|a_{1}\right|^{2}+\left|a_{2}\right|^{2}\right)-s_{1}\left(\left|b_{1}\right|^{2}+\left|b_{2}\right|^{2}\right) \xi m^{2}\right] \\
c_{2} & =-\frac{1}{16}\left[256 \frac{m_{*}^{2}}{m^{2}}\left(\left|a_{1}\right|^{2}+\left|a_{2}\right|^{2}\right)+s_{1}\left|a_{2} b_{1}-a_{1} b_{2}\right|^{2} \xi m^{2}\right] \\
c_{1} & =-s_{1} \frac{m_{*}^{2}}{m^{2}}\left|a_{2} b_{1}-a_{1} b_{2}\right|^{2}\left(\xi m^{2}\right)
\end{aligned}
$$

These expressions show explicitly the invariance of $c_{i}$ under unitary redefinitions of the $2 \times 2$ matrix of coefficients $a_{i}, b_{i}$, and this is a good consistency check. If $s_{1}>0$, there is one positive root and two negative. If $s_{1}<0$ we end up with two positive and one negative roots. For a unitary transformation in (9), the roots are

$$
\nu_{1,2}=\frac{1}{2}\left[1 \pm \sqrt{1+64 m_{*}^{2} / m^{2}}\right], \text { with } \nu_{1}>0 ; \quad \nu_{3}=-\frac{1}{16} s_{1} m^{2} \xi
$$

where as usual $m_{*}=\sqrt{\xi} m^{2} / 4$. We keep $m_{*}$ manifest in our equations in order to trace the effects of the initial constraint, eqs.(10) and (11). The Lagrangian becomes:

$$
\begin{aligned}
\mathcal{L}^{\prime} & =\int d^{4} \theta\left[\nu_{1} \Phi_{1}^{\prime \dagger} \Phi_{1}^{\prime}+\nu_{2} \Phi_{2}^{\prime \dagger} \Phi_{2}^{\prime}+\nu_{3} \Phi_{3}^{\prime \dagger} \Phi_{3}^{\prime}\right] \\
& +\left\{\int d^{2} \theta\left[\frac{1}{2} m_{k p} \Phi_{k}^{\prime} \Phi_{p}^{\prime}+\frac{1}{3} \lambda_{k p l} \Phi_{k}^{\prime} \Phi_{p}^{\prime} \Phi_{l}^{\prime}\right]+\text { h.c. }\right\}
\end{aligned}
$$

where $\Phi_{i}^{\prime}=v_{i j} \Phi_{j}, i, j=1,2,3$, and $\operatorname{diag}\left(\nu_{1}, \nu_{2}, \nu_{3}\right)=v \mathcal{M} v^{\dagger}$ with $v_{i j}$ unitary. Also

$$
\begin{aligned}
& m_{k p}=m\left(a_{1} v_{k 1}^{*}+a_{2} v_{k 2}^{*}\right)\left(a_{1} v_{p 1}^{*}+a_{2} v_{p 2}^{*}\right)-m_{*}\left(v_{k 3}^{*}\left(b_{1} v_{p 1}^{*}+b_{2} v_{p 2}^{*}\right)+(k \leftrightarrow p)\right) \\
& \lambda_{k p l}=\lambda\left(a_{1} v_{k 1}^{*}+a_{2} v_{k 2}^{*}\right)\left(a_{1} v_{p 1}^{*}+a_{2} v_{p 2}^{*}\right)\left(a_{1} v_{l 1}^{*}+a_{2} v_{l 2}^{*}\right)
\end{aligned}
$$

${ }^{8} \mathcal{M}$ has: $\mathcal{M}_{11}=\rho_{1}, \mathcal{M}_{12}=\rho_{2}, \mathcal{M}_{13}=\rho_{3}, \mathcal{M}_{21}=\rho_{2}^{*}, \mathcal{M}_{22}=\rho_{5}, \mathcal{M}_{23}=\rho_{4}, \mathcal{M}_{31}=\rho_{3}^{*}, \mathcal{M}_{32}=\rho_{4}^{*}, \mathcal{M}_{33}=0$ 
which are symmetric under the permutation of their indices. We rescale $\tilde{\Phi}_{i}^{\prime}$

$$
\Phi_{i}^{\prime}=\tilde{\Phi}_{i} / \sqrt{\left|\nu_{i}\right|}, \quad i=1,2,3
$$

to find:

$$
\begin{aligned}
\mathcal{L}^{\prime} & =\int d^{4} \theta\left[\sigma_{\nu_{1}} \tilde{\Phi}_{1}^{\dagger} \tilde{\Phi}_{1}+\sigma_{\nu_{2}} \tilde{\Phi}_{2}^{\dagger} \tilde{\Phi}_{2}+\sigma_{\nu_{3}} \tilde{\Phi}_{3}^{\dagger} \tilde{\Phi}_{3}\right] \\
& +\left\{\int d^{2} \theta\left[\frac{1}{2} \tilde{m}_{k p} \tilde{\Phi}_{k} \tilde{\Phi}_{p}+\frac{1}{3} \tilde{\lambda}_{k p l} \tilde{\Phi}_{k} \tilde{\Phi}_{p} \tilde{\Phi}_{l}\right]+\text { h.c. }\right\},
\end{aligned}
$$

where

$$
\sigma_{\nu_{k}}=\frac{\nu_{k}}{\left|\nu_{k}\right|}, \quad \tilde{m}_{k p}=\frac{m_{k p}}{\sqrt{\left|\nu_{k} \nu_{p}\right|}}, \quad \tilde{\lambda}_{k p l}=\frac{\lambda_{k p l}}{\sqrt{\left|\nu_{k} \nu_{p} \nu_{l}\right|}}, \quad k, p, l=1,2,3 .
$$

Therefore $\sigma_{\nu_{1}}=1, \sigma_{\nu_{2}}=-1, \sigma_{\nu_{3}}=-s_{1}$ and as a result $\tilde{\Phi}_{1}$ is a particle-like field, $\tilde{\Phi}_{2}$ and $\tilde{\Phi}_{3}$ (for $s_{1}=1$ ) are ghost-like superfields given their negative kinetic terms. The presence of such superfields is common in supersymmetric theories with constraints [45].

The result in eq.(22) shows that we "unfolded" in a manifest supersymmetric way the original, higher derivative supersymmetric Lagrangian $\mathcal{L}$ eqs.(8) into an equivalent, second order Lagrangian. As a result, while in the initial (8) the auxiliary field $F$ was dynamical, all new $\tilde{F}_{i}$ in $\mathcal{L}^{\prime}$ are not and can be integrated out as usual. To understand this change, recall from (14) the components of $\Phi_{D} \equiv\left(\phi_{D}, \psi_{D}, F_{D}\right) \sim\left(-4 F^{*} ;-4 i \not \bar{\psi} ; 4 \square \phi^{*}\right)$; further, original $\left(\Phi, \Phi_{D}\right)$ were traded for $\left(\Phi_{1}, \Phi_{2}\right) \rightarrow\left(\tilde{\Phi}_{1}, \tilde{\Phi}_{2}\right)$; using these components of $\Phi_{D}$ we see then that $\tilde{\Phi}_{2}^{\dagger} \tilde{\Phi}_{2}$ in (22) accounts for the kinetic term of original $F$ and for the higher derivative terms for original $\psi, \phi$ in (8) . However, since each of the components of $\Phi_{D}$ were not independent of those of $\Phi$, a constraint had to account for this, which was "traded" for a new chiral superfield $\tilde{\Phi}_{3}$. In this case $\tilde{\Phi}_{3}$ is dynamical, but we shall see in Section 4 that this is not always the case 9 . Further

$$
\sigma_{\nu_{j}} \tilde{F}_{j}^{*}=-\left[\tilde{m}_{k j} \tilde{\phi}_{k}+\tilde{\lambda}_{k p j} \tilde{\phi}_{k} \tilde{\phi}_{p}\right], \quad j=1,2,3 .
$$

\footnotetext{
${ }^{9}$ It is perhaps useful to mention here the non-susy situation, discussed long ago in [41]. In this case taking $L=-1 / 2 \phi\left(\square+\xi \square^{2}\right) \phi$, and after introducing the lagrangian multiplier $\lambda$ one finds $L^{\prime}=-1 / 2 \phi \rho-1 / 2 \xi \rho^{2}+$ $\lambda(\square \phi-\rho)$ thus the field $\rho$ is not dynamical and can be integrated out (alternatively one can factorise the " $\square$ " dependence in $L$ to end up with two dynamical fields [41]). Therefore only two fields are present in the end, $\phi$ and $\lambda$. This is different from the supersymmetric case in the text where original $F$ was dynamical, which by supersymmetry required the introduction of an additional (third) (super)field in the second order formulation.
} 
The scalar potential of the "unfolded" theory is 10 :

$$
V=\sigma_{\nu_{j}}\left|\tilde{F}_{j}\right|^{2}=\sigma_{\nu_{j}}\left|\tilde{m}_{k j} \tilde{\phi}_{k}+\tilde{\lambda}_{k p j} \tilde{\phi}_{k} \tilde{\phi}_{p}\right|^{2} \quad k, p, j=1,2,3
$$

Therefore the scalar potential $\mathrm{V}$ is not positive definite in the second order theory; it has contributions which are negative due to ghost superfields.

\subsection{The mass spectrum.}

Let us investigate the mass spectrum. One obtains for the trilinear couplings (20)

$$
\tilde{\lambda}_{111}=\tilde{\lambda}_{221}=-\tilde{\lambda}_{112}=-\tilde{\lambda}_{222}=-\frac{\lambda}{\eta^{3 / 4}}, \quad \tilde{\lambda}_{i j 3}=0, \quad i, j=1,2,3,
$$

symmetric under a permutation of their indices; also

$$
\eta \equiv 1+64 m_{*}^{2} / m^{2}
$$

For the bilinear couplings

$$
\begin{array}{ll}
\tilde{m}_{11}=\tilde{m}_{22}=-\tilde{m}_{12}=\frac{m}{\sqrt{\eta}}, & \tilde{m}_{33}=0 \\
\tilde{m}_{13}=\frac{1-\sqrt{\eta}}{2 \sqrt{\xi} \eta^{1 / 4}}, & \tilde{m}_{23}=-\frac{1+\sqrt{\eta}}{2 \sqrt{\xi} \eta^{1 / 4}},
\end{array}
$$

Note that $m_{23} \sim-1 / \sqrt{\xi}$ while the rest are finite when $\xi \rightarrow 0$ (recalling that $m_{*}=m^{2} \sqrt{\xi} / 4$ ). With the above relations, the Lagrangian simplifies into

$$
\begin{aligned}
\mathcal{L}^{\prime} & =\int d^{4} \theta\left[\tilde{\Phi}_{1}^{\dagger} \tilde{\Phi}_{1}-\tilde{\Phi}_{2}^{\dagger} \tilde{\Phi}_{2}-s_{1} \tilde{\Phi}_{3}^{\dagger} \tilde{\Phi}_{3}\right] \\
& +\left\{\int d^{2} \theta\left[\left(\tilde{m}_{13} \tilde{\Phi}_{1}+\tilde{m}_{23} \tilde{\Phi}_{2}\right) \tilde{\Phi}_{3}+\frac{\tilde{m}_{11}}{2}\left(\tilde{\Phi}_{1}-\tilde{\Phi}_{2}\right)^{2}+\frac{\tilde{\lambda}_{111}}{3}\left(\tilde{\Phi}_{1}-\tilde{\Phi}_{2}\right)^{3}\right]+\text { h.c. }\right\}
\end{aligned}
$$

which shows that $\tilde{\Phi}_{3}$ has no trilinear interaction.

The tree-level couplings $\left(\tilde{m}_{i j}\right.$ and $\left.\tilde{\lambda}_{i j k}\right)$ of the new theory have acquired a scale (moduli) dependence. Here we refer to their dependence on $\xi$ which is explicit in eqs.(28) and to that

\footnotetext{
${ }^{10}$ Sums over repeating indices are understood.

${ }^{11}$ For $m_{13}$ and $m_{23}$ one also obtains an overall phase factor $\exp \left(-i h_{1}\right) \operatorname{sign}(\sec \theta)$ multiplying the values shown in (28), and which was not written there since it can be absorbed into a redefinition of $\tilde{\Phi}_{3}$, see (29).
} 
induced via $m_{*} \sim \sqrt{\xi}$, where $m_{*}$ is the parameter controlling the presence of the constraint $\Delta \mathcal{L}$, eqs.(11), (27). Therefore the constraint itself introduced dynamically a scale dependence of the couplings. This dependence is ultimately due to the higher derivative operators (8) whose initial presence in the Lagrangian was "traded" for a threshold correction to the couplings of the Lagrangian of the second order theory.

The relation of initial fields $\Phi, \Phi_{D}$ to the new basis is, for the unitary transformation (9)

$$
\Phi=\frac{1}{\eta^{1 / 4}}\left(\tilde{\Phi}_{2}-\tilde{\Phi}_{1}\right), \quad \Phi_{D}=\frac{4}{m \sqrt{\xi}} \tilde{\Phi}_{3}
$$

This shows that the original superfield $\Phi$ has actually a "ghost-like" component $\left(\tilde{\Phi}_{2}\right)$; note that the overall factor $\eta$ depends on the scale of the higher derivative operator.

From (29) one finds the scalar potential:

$$
\begin{aligned}
V & =\left|\tilde{F}_{1}\right|^{2}-\left|\tilde{F}_{2}\right|^{2}-s_{1}\left|\tilde{F}_{3}\right|^{2} \\
& =\left(\tilde{m}_{13}^{2}-\tilde{m}_{23}^{2}\right)\left|\tilde{\phi}_{3}\right|^{2}+\left(\tilde{m}_{13}+\tilde{m}_{23}\right)\left\{\left[\tilde{\phi}_{3}^{*}\left(\tilde{m}_{11} \tilde{\phi}_{-}+\tilde{\lambda}_{111} \tilde{\phi}_{-}^{2}\right)\right]+\text { c.c. }\right\} \\
& -s_{1}\left|\tilde{m}_{13} \tilde{\phi}_{1}+\tilde{m}_{23} \tilde{\phi}_{2}\right|^{2}
\end{aligned}
$$

The quartic interaction is not present anymore in the potential of the "unfolded" Lagrangian, which only contains bilinear and cubic terms. Tree-level quartic interactions are nevertheless generated in the low energy limit by exchange of $\tilde{\phi}_{3}$. This form of the potential seems unstable due to cubic terms present and would suggest that such stability be only addressed locally (i.e. we demand that $\xi \tilde{\phi}_{i}^{2} \ll 1$ ) and other higher dimensional operators of similar order could affect the Lagrangian at large $\tilde{\phi}_{i}$. In fact, the discussion of stability in ghost directions is rather subtle as we shall see later, due to the fact that these fields have negative kinetic terms. We return to clarify this shortly. The vanishing of the first derivatives wrt $\tilde{\phi}_{1,2,3}^{*}$ respectively, gives

$$
\begin{aligned}
& \left\langle\tilde{\phi}_{3}\right\rangle=\frac{-1}{\tilde{m}_{13}-\tilde{m}_{23}}\left[\tilde{m}_{11}+\tilde{\lambda}_{111}\left(\left\langle\tilde{\phi}_{1}\right\rangle-\left\langle\tilde{\phi}_{2}\right\rangle\right)\right]\left(\left\langle\tilde{\phi}_{1}\right\rangle-\left\langle\tilde{\phi}_{2}\right\rangle\right) \\
& \tilde{m}_{13}\left\langle\tilde{\phi}_{1}\right\rangle+\tilde{m}_{23}\left\langle\tilde{\phi}_{2}\right\rangle=0, \quad \text { and }
\end{aligned}
$$

i) $\quad\left\langle\tilde{\phi}_{1}\right\rangle-\left\langle\tilde{\phi}_{2}\right\rangle=0, \quad$ or $\quad$ ii $)\left\langle\tilde{\phi}_{1}\right\rangle-\left\langle\tilde{\phi}_{2}\right\rangle=-\frac{\tilde{m}_{11}}{\tilde{\lambda}_{111}}, \quad$ or $\quad$ iii $)\left\langle\tilde{\phi}_{1}\right\rangle-\left\langle\tilde{\phi}_{2}\right\rangle=-\frac{\tilde{m}_{11}}{2 \tilde{\lambda}_{111}}(34)$ 
These cases are discussed below. In case $i$ ) we have, using (32), (33):

$$
\left\langle\tilde{F}_{1,2,3}\right\rangle=0, \quad\left\langle\tilde{\phi}_{1,2,3}\right\rangle=0, \quad V_{*}=0
$$

where $V_{*}$ denotes the value of the scalar potential at this extremum point. One computes the eigenvalues of the mass matrix of second derivatives of the scalar potential $V$, which is expanded about this vacuum solution, in the basis $\left(\tilde{\phi}_{i}, \tilde{\phi}_{i}^{*}\right), i=1,2,3$. At this extremum point one finds (for $s_{1}=+1$ )

$$
\begin{aligned}
m_{\tilde{\phi}_{1}}^{2} & =m^{2}\left[1-7\left(\xi m^{2}\right)^{2}+\mathcal{O}\left(\left(\xi m^{2}\right)^{5 / 2}\right)\right] \\
m_{\tilde{\phi}_{2,3}}^{2} & =m^{2}\left[-\frac{1}{\xi m^{2}} \mp \frac{1}{\sqrt{\xi} m}-\frac{1}{2} \pm \frac{19 \sqrt{\xi} m}{8}+\mathcal{O}\left(\xi m^{2}\right)\right]
\end{aligned}
$$

where either the upper or lower signs are to be considered, for $\tilde{\phi}_{2,3}$ respectively. The first eigenvalue should correspond to our original $\phi$ in (6), (8) of mass $m$, for the same supersymmetric state. There are also two negative mass eigenstates corresponding to the two ghost superfields present in the "unfolded" Lagrangian of second order. Their negative signs are expected since the kinetic and mass terms of ghost superfields come with opposite sign in the action and thus do not necessarily suggest an instability of the potential in the vicinity of this vacuum. There is however a problem. The above spectrum is different from that in (77) of the original Lagrangian (6), although the latter is equivalent to that in (29) if the two formulations are indeed equivalent, as showed. What is, then, the origin of this discrepancy?

To understand this, note that - unlike above - one should compute the mass eigenvalues from the potential with a metric which takes account of the different sign of the ghosts' kinetic terms. For this one goes to the basis $\phi_{i}=\left(a_{i}+i b_{i}\right) / \sqrt{2}, \phi_{i}^{*}=\left(a_{i}-i b_{i}\right) / \sqrt{2}$ where $a_{i}, b_{i}(i=1,2,3)$ are real components, then rescale $a_{i}, b_{i}$ for $i=2,3$, into $a_{i} \rightarrow i a_{i}, b_{i} \rightarrow i b_{i}$, $\left(a_{1}, b_{1}\right.$ fixed). This rescaling ensures positive definite kinetic terms for the ghost terms. In the basis $\left(a_{1}, b_{1}, i a_{2}, i b_{2}, i a_{3}, i b_{3}\right)$ any negative eigenvalue of the mass matrix will signal a local instability. In this new basis the matrix of second derivatives of the potential has eigenvalues controlled by the characteristic equation

$$
\left(\square(1+\xi \square)^{2}+m^{2}\right)=0
$$

which is identical to that discussed in the text after eq.(66). Therefore, the spectrum of the original Lagrangian (6), (8) of the theory with higher derivative operators is indeed identical 
to that of the second order theory, computed after an appropriate rescaling of their real components, to account for their initial negative kinetic terms. This is a good check of the equivalence of the two formulations of the theory, eqs.(6) and (29), and of the introduction of the additional constraint superfield in (111). It also shows explicitly the requirement for using a different field-space metric when computing the spectrum in a second order theory with ghost fields. These observations fix the problem mentioned above and invalidate (36). The correct spectrum is then

$$
\begin{aligned}
m_{\tilde{\tilde{\phi}}_{1}}^{2} & =m^{2}\left[1+2 \xi m^{2}+7\left(\xi m^{2}\right)^{2}+\mathcal{O}\left(\left(\xi m^{2}\right)^{5 / 2}\right)\right] \\
m_{\tilde{\tilde{\phi}}_{2,3}}^{2} & =m^{2}\left[\frac{1}{\xi m^{2}} \pm \frac{1}{\sqrt{\xi m^{2}}}-\frac{1}{2} \pm \frac{5 \sqrt{\xi m^{2}}}{8}-\xi m^{2}+\mathcal{O}\left(\left(\xi m^{2}\right)^{3 / 2}\right)\right]
\end{aligned}
$$

in agreement with (7). In the basis of rescaled component fields of positive definite kinetic terms all mass eigenvalues are positive and this vacuum is therefore stable.

In case $i i)$, using (32), (33):

$$
\left\langle\tilde{F}_{1,2,3}\right\rangle=0, \quad\left\langle\tilde{\phi}_{1}\right\rangle=\frac{m(1+\sqrt{\eta})}{2 \eta^{1 / 4} \lambda}, \quad\left\langle\tilde{\phi}_{2}\right\rangle=\frac{m(1-\sqrt{\eta})}{2 \eta^{1 / 4} \lambda}, \quad\left\langle\tilde{\phi}_{3}\right\rangle=0, \quad V_{*}=0
$$

with the vev of $\tilde{\phi}_{2}$ going to 0 if $\xi m^{2} \rightarrow 0$. In this case the eigenvalues of the matrix of second derivatives of the scalar potential are identical to those of case i), eq.(38). The same situation as in $i$ ) applies regarding the stability. Supersymmetry is unbroken in both $i$ ) and $i i$ ), and in the limit $\xi \rightarrow 0$ one recovers from $i$ ), ii) the two supersymmetric ground states of the Wess-Zumino models without higher dimensional operators.

Finally, consider case iii) together with (32), (33). One obtains

$$
\left\langle\tilde{F}_{1}^{*}\right\rangle=-\frac{m^{2}(1+\sqrt{\eta})}{8 \lambda \eta^{1 / 4}}, \quad\left\langle\tilde{F}_{2}^{*}\right\rangle=-\frac{m^{2}(1-\sqrt{\eta})}{8 \lambda \eta^{1 / 4}}, \quad\left\langle\tilde{F}_{3}\right\rangle=0
$$

and

$$
\left\langle\tilde{\phi}_{1}\right\rangle=\frac{m(1+\sqrt{\eta})}{4 \eta^{1 / 4} \lambda}, \quad\left\langle\tilde{\phi}_{2}\right\rangle=\frac{m(1-\sqrt{\eta})}{4 \eta^{1 / 4} \lambda}, \quad\left\langle\tilde{\phi}_{3}\right\rangle=-\frac{m^{2} \sqrt{\xi}}{4 \lambda}, \quad V_{*}=\frac{m^{4}}{16 \lambda^{2}}
$$

The vev's of $\tilde{\phi}_{2,3}$ vanish when $\xi m^{2} \rightarrow 0$. In terms of the component fields of the original Lagrangian (8), the values (40) correspond to $F=m^{2} / 4 \lambda$. Therefore, as expected $V_{*}=F^{2}$; this is a check of above results since the value of the potential at an extremum point does 
not depend on the dynamical nature of the original $F$. These relations are easily seen to be consistent with the relation between $\tilde{\Phi}_{D}, \tilde{\Phi}$ and new $\tilde{\Phi}_{i}$ see (30) (and also (9), (14)).

Next we compute the matrix of second derivatives of $V$ and its eigenvalues at this extremum point. As discussed, we use a diagonalisation method which takes into account the negative signature of the kinetic terms of the two ghosts fields. We find

$$
\begin{array}{rlrl}
m_{\tilde{\phi}_{1}}^{2}=\frac{-m^{2}}{1+\sqrt{1+2 \xi m^{2}}} & m_{\tilde{\phi}_{2}}^{2}=\frac{m^{2}}{1+\sqrt{1-2 \xi m^{2}}} \\
m_{\tilde{\tilde{\phi}}_{3}}^{2}=\frac{m^{2}}{-1+\sqrt{1+2 \xi m^{2}}}, & m_{\tilde{\tilde{\phi}}_{4}}^{2}=\frac{m^{2}}{1-\sqrt{1-2 \xi m^{2}}}, & m_{\tilde{\phi}_{5,6}}^{2}=\frac{1}{\xi}
\end{array}
$$

The values $m_{\tilde{\tilde{\phi}}_{3,4,5,6}}^{2}$ are now all positive, and do not suggest a local instability. The signs of $m_{\tilde{\tilde{\phi}} 1,2}^{2} \approx \mp m^{2} / 2$ are independent of the rescaling of the ghosts real component fields. Finally, $m_{\tilde{\phi}_{1,2}}^{\stackrel{\phi_{1,2}}{2}}$ are counterparts to those at the saddle point of $V$ in the absence of the higher derivative term, given by $\pm m^{2} / 2$, for same corresponding vev of $\tilde{\phi}_{1,2}$ and $\phi$ respectively (see section 3.1). Due to non-zero vev's of the auxiliary fields, supersymmetry is in this case broken, similarly to Wess-Zumino model without higher dimensional operators. This ends our discussion on the spectrum obtained from the second order Lagrangian.

There remains the question of the relation between the potential $V$ in (31) and that of the original theory (8) and how the latter is recovered from the former in the limit $12 \xi \rightarrow 0$. To this purpose, evaluate $V$ of (31) for extremum vev's given in eqs.(32), (33) but not in (34). The value obtained is

$$
V_{*}^{\prime}=\sqrt{\eta}\left|\tilde{\lambda}_{111}\left\langle\tilde{\phi}_{-}\right\rangle^{2}+\tilde{m}_{11}\left\langle\tilde{\phi}_{-}\right\rangle\right|^{2}=\left|\lambda \eta^{-\frac{1}{2}}\left\langle\tilde{\phi}_{-}\right\rangle^{2}-m \eta^{-\frac{1}{4}}\left\langle\tilde{\phi}_{-}\right\rangle\right|^{2}
$$

with $\tilde{\phi}_{-} \equiv \tilde{\phi}_{1}-\tilde{\phi}_{2} . V_{*}^{\prime}$ is thus the value of $V$ evaluated at extremum vev's in directions other than $\tilde{\phi}_{1}-\tilde{\phi}_{2}$. One observes that the extremum condition on $V_{*}^{\prime}$ with respect to "variable" $\left\langle\tilde{\phi}_{-}\right\rangle \equiv\left\langle\tilde{\phi}_{1}-\tilde{\phi}_{2}\right\rangle$ recovers the remaining condition eq.(34) of those in (32) to (34). On $V_{*}^{\prime}$ the limit $\xi \rightarrow 0$ is well defined and finite. Recalling the scalar potential of the original theory

$$
V=\left|\lambda \phi^{2}+m \phi\right|^{2}, \quad \text { and } \quad \phi=-\frac{1}{\eta^{1 / 4}}\left(\tilde{\phi}_{1}-\tilde{\phi}_{2}\right)
$$

one recovers eqs.(43). To conclude, the scalar potential in the original theory (8) is a "projection" of a more general potential which includes the extra (ghost) degrees of freedom

\footnotetext{
${ }^{12}$ Eq.(21) is singular if $\xi \rightarrow 0, \nu_{2,3} \rightarrow 0$; in (31) $m_{13}^{2}-m_{23}^{2}=-1 / \xi, m_{13}+m_{23} \sim-1 / \sqrt{\xi}$ are singular too.
} 
introduced by the higher derivative term in the action, evaluated for extremum vev's of two linear combinations of all degrees of freedom. That is, there is no clear separation in the potential between particle and ghost directions. This is not too surprising if we recall eq.(30) showing the original $\Phi$ had itself a ghost "piece".

\subsection{The case of a general superpotential.}

We extend the results of the previous section to the case of an arbitrary superpotential $W(\Phi, S)$, which can include higher dimensional operators, but no higher derivative term 13 . One can also have additional superfields, generically denoted here $S$, with standard kinetic terms. Consider

$$
\mathcal{L}=\int d^{4} \theta\left[\Phi^{\dagger}(1+\xi \square) \Phi+S^{\dagger} S\right]+\left\{\int d^{2} \theta W(\Phi, S)+\text { h.c. }\right\}
$$

Following steps similar to those in the previous section, $\mathcal{L}$ is shown to be equivalent to

$$
\begin{aligned}
\mathcal{L}^{\prime} & =\int d^{4} \theta\left[\tilde{\Phi}_{1}^{\dagger} \tilde{\Phi}_{1}-\tilde{\Phi}_{2}^{\dagger} \tilde{\Phi}_{2}-\tilde{\Phi}_{3}^{\dagger} \tilde{\Phi}_{3}+S^{\dagger} S\right] \\
& +\left\{\int d^{2} \theta\left[\frac{1}{2} \tilde{\mu}_{k p} \tilde{\Phi}_{k} \tilde{\Phi}_{p}+W\left[\Phi\left(\tilde{\Phi}_{1,2,3}\right), S\right]\right]+\text { h.c. }\right\},
\end{aligned}
$$

with the following relations:

$$
\Phi\left(\tilde{\Phi}_{1,2,3}\right)=\frac{1}{\eta^{1 / 4}}\left[\left(\tilde{\Phi}_{2}-\tilde{\Phi}_{1}\right)\right], \quad \Phi_{D}=\frac{4}{m \sqrt{\xi}} \tilde{\Phi}_{3}
$$

Notice that $S$ is spectator under going from the fourth order to second order theory, since it has no higher derivative kinetic terms and does not mix with the kinetic terms of $\Phi$. One also finds that $\tilde{\mu}_{i j}=0$ for all $i, j$ except:

$$
\tilde{\mu}_{13}=\tilde{\mu}_{31}=\frac{1-\sqrt{\eta}}{2 \eta^{1 / 4} \sqrt{\xi}}, \quad \tilde{\mu}_{23}=\tilde{\mu}_{32}=-\frac{1+\sqrt{\eta}}{2 \eta^{1 / 4} \sqrt{\xi}}
$$

where as usual $\eta=1+4 \xi m^{2}$. These values are equal to $\tilde{m}_{13}, \tilde{m}_{23}$ of (28) and are generated by the constraint (11) alone, and not by bilinears that may be present in $W$. Also $\tilde{\mu}_{13}=\tilde{\mu}_{31} \approx 0$, $\tilde{\mu}_{23}=\tilde{\mu}_{32} \approx-1 / \sqrt{\xi}$ for $\xi m^{2} \ll 1$. The auxiliary fields are

\footnotetext{
${ }^{13}$ This case is discussed in Section 4

${ }^{14}$ If $S$ itself has higher derivative kinetic terms one introduces extra constraint superfields see previous section.
} 


$$
\begin{aligned}
\tilde{F}_{1}^{*} & =-\tilde{\mu}_{31} \tilde{\phi}_{3}+\eta^{-1 / 4} W_{\phi}^{\prime} \\
-F_{2}^{*} & =-\tilde{\mu}_{32} \tilde{\phi}_{3}-\eta^{-1 / 4} W_{\phi}^{\prime} \\
-F_{3}^{*} & =-\tilde{\mu}_{k 3} \tilde{\phi}_{k}, \quad F_{S}^{*}=-W_{\phi_{s}}^{\prime}
\end{aligned}
$$

Here $W_{\phi}^{\prime} \equiv \partial W\left(\phi, \phi_{S}\right) / \partial \phi$, with $\phi$ replaced by $\phi=\phi\left(\tilde{\phi}_{1,2}\right)$ of (47). The scalar potential becomes

$$
V=\left|\tilde{\mu}_{31} \tilde{\phi}_{3}-\eta^{-1 / 4} W_{\phi}^{\prime}\right|^{2}-\left|\tilde{\mu}_{32} \tilde{\phi}_{3}+\eta^{-1 / 4} W_{\phi}^{\prime}\right|^{2}-\left|\tilde{\mu}_{k 3} \tilde{\phi}_{k}\right|^{2}+\left|W_{\phi_{s}}^{\prime}\right|^{2}
$$

In the original $\Phi$-dependent formulation, the potential which was function of $F, \phi, F_{s}, \phi_{s}$, evaluated at an extremum point(s) labelled by "o", was

$$
V_{o}=\left[\left|W_{\phi}^{\prime}\right|^{2}+\left|W_{\phi_{s}}^{\prime}\right|^{2}\right]_{o}
$$

Let us assume that supersymmetry is unbroken. In the original-field language this means that $W_{\phi}^{\prime}=W_{\phi_{s}}^{\prime}=0$ and also $F=F_{s}=0$ at this extremum point. This is true regardless of the dynamical nature of $F$. Let us now investigate the corresponding situation in the second order theory. The extremum conditions for (50), $\partial V / \partial \tilde{\phi}_{p}=0, p=1,2,3$ give, at the extremum point considered:

$$
\left\langle\tilde{\phi}_{3}\right\rangle=0, \quad\left\langle\tilde{\mu}_{3 k} \tilde{\phi}_{k}\right\rangle=0, \quad k=1,2 .
$$

From (49), at the extremum point of the scalar potential we find $\tilde{F}_{i}=F_{s}=0, i=1,2,3$. The vanishing of the auxiliary fields of the second order theory confirms, in the new formalism, that supersymmetry is unbroken in this state, as the equivalence of the two formulations of the theory would suggest.

\section{Effects of higher derivatives in the superpotential}

The method developed so far can be applied when higher derivative terms are present in the superpotential. Consider the Lagrangian

$$
\begin{aligned}
\mathcal{L} & =\int d^{4} \theta \Phi^{\dagger} \Phi+\left\{\int d^{2} \theta\left[s_{2} \sqrt{\xi} \Phi \square \Phi+\frac{m}{2} \Phi^{2}+\frac{\lambda}{3} \Phi^{3}\right]+\text { h.c. }\right\} \\
& =\int d^{4} \theta\left[\Phi^{\dagger} \Phi+\frac{s_{2} \sqrt{\xi}}{4}\left(\Phi D^{2} \Phi+\Phi^{\dagger} \bar{D}^{2} \Phi^{\dagger}\right)\right]+\left\{\int d^{2} \theta\left[\frac{m}{2} \Phi^{2}+\frac{\lambda}{3} \Phi^{3}\right]+\text { h.c. }\right\}
\end{aligned}
$$


where 15 we allow $s_{2}= \pm 1$. We follow the steps of the previous section, introduce (9), a Lagrange multiplier chiral superfield and $\Delta \mathcal{L}$, eqs.(10), (11). The counterpart to eqs.(16) has now the roots

$$
\nu_{1,2}=\frac{1}{2}[1 \pm \sqrt{\eta} \prime], \quad \nu_{3}=0, \quad \text { where } \quad \eta^{\prime} \equiv 1+4 m^{2} \xi\left(1+s_{2}^{2} / 16\right)
$$

with the choice $\nu_{1}>0$. Unlike in eq.(18), there is a vanishing eigenvalue, so there is one ghost superfield and one particle-like superfield. After appropriate normalisation of the Kahler terms, the Lagrangian equivalent to that in (53) is:

$$
\mathcal{L}^{\prime}=\int d^{4} \theta\left[\tilde{\Phi}_{1}^{\dagger} \tilde{\Phi}_{1}-\tilde{\Phi}_{2}^{\dagger} \tilde{\Phi}_{2}\right]+\left\{\int d^{2} \theta\left[\frac{1}{2} \tilde{m}_{k p} \tilde{\Phi}_{k} \tilde{\Phi}_{p}+\frac{1}{3} \tilde{\lambda}_{k p l} \tilde{\Phi}_{k} \tilde{\Phi}_{p} \tilde{\Phi}_{l}\right]+\text { h.c. }\right\},
$$

where

$$
\tilde{m}_{k p}=\frac{m_{k p}}{\sqrt{\left|\nu_{k}^{q_{k 3}} \nu_{p}^{q_{p 3}}\right|}}, \quad \tilde{\lambda}_{k p l}=\frac{\lambda_{k p l}}{\sqrt{\mid \nu_{k}^{q_{k 3}} \nu_{p}^{q_{p 3}} \nu_{l}^{q_{l 3} \mid}}}, \quad k, p, l=1,2,3
$$

with $q_{k 3}=1-\delta_{k 3}$, and $\tilde{m}_{k p}, \tilde{\lambda}_{k p l}$ are given in (20) with $v_{i j}$ presented in the Appendix, eq. (89). As before $\tilde{\lambda}_{i j 3}=0 i, j=1,2,3$. $\tilde{\Phi}_{3}$ can be eliminated using the equations of motion:

$$
\tilde{m}_{k 3} \tilde{\Phi}_{k}=0, \quad \tilde{\Phi}_{3}=-\frac{1}{\tilde{m}_{33}}\left(\tilde{m}_{13} \tilde{\Phi}_{1}+\tilde{m}_{23} \tilde{\Phi}_{2}\right)
$$

Unlike in Section [3, here $\tilde{\Phi}_{3}$ can be eliminated, and this is ultimately due to the fact that in (53) $F$ is not dynamical and can be integrated out. As a result $\mathcal{L}^{\prime}$ can be re-written

$$
\begin{aligned}
\mathcal{L}^{\prime} & =\int d^{4} \theta\left[\tilde{\Phi}_{1}^{\dagger} \tilde{\Phi}_{1}-\tilde{\Phi}_{2}^{\dagger} \tilde{\Phi}_{2}\right] \\
+ & \left\{\int d^{2} \theta\left[\frac{1}{2}\left(d_{1} \tilde{\Phi}_{1}^{2}+d_{2} \tilde{\Phi}_{2}^{2}+2 d_{3} \tilde{\Phi}_{1} \tilde{\Phi}_{2}\right)+\frac{\tilde{\mu}}{2}\left(\tilde{\Phi}_{1}-\tilde{\Phi}_{2}\right)^{2}+\frac{\tilde{\lambda}_{111}}{3}\left(\tilde{\Phi}_{1}-\tilde{\Phi}_{2}\right)^{3}\right]+\text { h.c. }\right\}
\end{aligned}
$$

where

$$
\tilde{\mu}=\frac{m}{\sqrt{\eta^{\prime}}}, \quad \tilde{\lambda}_{111}=-\frac{\lambda}{\eta^{\prime 3 / 4}}
$$

and

$$
d_{1}=\frac{\left(\sqrt{\eta^{\prime}}-1\right)^{2}}{8 s_{2} \sqrt{\eta^{\prime} \xi}}, \quad d_{2}=\frac{\left(\sqrt{\eta^{\prime}}+1\right)^{2}}{8 s_{2} \sqrt{\eta^{\prime} \xi}}, \quad d_{3}=\frac{\eta^{\prime}-1}{8 s_{2} \sqrt{\eta^{\prime} \xi}}
$$

with $d_{1}=\mathcal{O}\left(\xi^{3 / 2} m^{4}\right)$ and $d_{3}=\mathcal{O}\left(\sqrt{\xi} m^{2}\right)$ vanishing in the limit of small $\xi m^{2}$; finally $d_{2}=$ $\mathcal{O}(1 / \sqrt{\xi})$ gives the leading contribution to the mass of the ghost superfield $16 \tilde{\Phi}_{2}$.

\footnotetext{
${ }^{15}$ We used $\int d^{4} x d^{2} \theta \bar{D}^{2} Q(x, \theta, \bar{\theta})=-4 \int d^{4} x d^{4} \theta Q(x, \theta, \bar{\theta})$.

${ }^{16}$ The whole field-dependent term involving coefficients $d_{1,2,3}$ equals to $\left(s_{2} / 16\right) \sqrt{\xi} \Phi_{D}^{2}$ with $\Phi_{D}$ of (61), (99).
} 
One also finds the relation between old and new fields:

$$
\Phi=\frac{1}{\eta^{\prime 1 / 4}}\left[\tilde{\Phi}_{2}-\tilde{\Phi}_{1}\right], \quad \Phi_{D}=\frac{1}{\eta^{1 / 4} \sqrt{\xi} s_{2}}\left[\left(1-\sqrt{\eta^{\prime}}\right) \tilde{\Phi}_{1}-\left(1+\sqrt{\eta^{\prime}}\right) \tilde{\Phi}_{2}\right]
$$

with $\Phi_{D}$ introduced in (99). Eqs.(59), (60) show again that the couplings of the second order theory acquired a scale $(\xi)$ dependence, via fields rescaling, first eq in (61).

The scalar potential is

$$
\begin{aligned}
V & =\left|\tilde{F}_{1}\right|^{2}-\left|\tilde{F}_{2}\right|^{2} \\
& =\left|\tilde{\mu}\left(\tilde{\phi}_{1}-\tilde{\phi}_{2}\right)+\tilde{\lambda}_{111}\left(\tilde{\phi}_{1}-\tilde{\phi}_{2}\right)^{2}+d_{1} \tilde{\phi}_{1}+d_{3} \tilde{\phi}_{2}\right|^{2} \\
& -\left|\tilde{\mu}\left(\tilde{\phi}_{1}-\tilde{\phi}_{2}\right)+\tilde{\lambda}_{111}\left(\tilde{\phi}_{1}-\tilde{\phi}_{2}\right)^{2}-d_{3} \tilde{\phi}_{1}-d_{2} \tilde{\phi}_{2}\right|^{2}
\end{aligned}
$$

In the basis of the second order theory, the potential is not positive definite anymore, similarly to the previous section. There are two ground states, for

$$
\begin{aligned}
& \text { i) }\left\langle\tilde{\phi}_{1}\right\rangle=\left\langle\tilde{\phi}_{2}\right\rangle=0 \\
& \text { ii) }\left\langle\tilde{\phi}_{1}\right\rangle=\frac{m\left(1+\sqrt{\eta^{\prime}}\right)}{2 \eta^{\prime 1 / 4} \lambda}, \quad\left\langle\tilde{\phi}_{2}\right\rangle=\frac{m\left(1-\sqrt{\eta^{\prime}}\right)}{2 \eta^{\prime 1 / 4} \lambda}
\end{aligned}
$$

which are similar to their counterparts in (35), (391). The vev's above give that $\left(\left\langle\tilde{\phi}_{2}\right\rangle-\right.$ $\left.\left\langle\tilde{\phi}_{1}\right\rangle\right) / \eta^{\prime 1 / 4}$ equals 0 for $i$ ), and $-m / \lambda$ for $\left.i i\right)$. This result for the two ground states is in agreement with what one obtains in the Wess-Zumino model in the absence of higher derivative operators, using the first relation in (61), for the corresponding ground states $\langle\phi\rangle=0$ and $-m / \lambda$, see section 3.1. In the limit of decoupling the higher dimensional operator $\xi \rightarrow 0$ then $\eta^{\prime} \rightarrow 1,\left\langle\tilde{\phi}_{2}\right\rangle=0$ and $-\left\langle\tilde{\phi}_{1}\right\rangle \rightarrow\langle\phi\rangle$. The ghost (super)field decouples and one recovers the Wess-Zumino model without higher derivatives. For both $i$ ) and $i i)$ cases:

$$
\left\langle\tilde{F}_{1}\right\rangle=\left\langle\tilde{F}_{2}\right\rangle=0, \quad V_{*}=0
$$

i.e. supersymmetry is unbroken, in agreement with the picture in the original basis for the corresponding ground states. One then computes the spectrum for i), ii), in basis $\tilde{\phi}_{1,2}$ with a metric which takes account of the negative sign of the kinetic term of $\tilde{\Phi}_{2}$, similar to the previous section. The solutions are (with fixed $s_{2}= \pm 1$ ): 


$$
m_{\tilde{\tilde{\phi}}_{1,2}}^{2}=\frac{1}{8 \xi}\left[1 \mp \sqrt{1+8 m s_{2} \sqrt{\xi}}+4 m s_{2} \sqrt{\xi}\right] .
$$

These values agree with those obtained from the poles of scalar propagators found using the old basis $(\phi, F)$ after performing the Grassmann integrals in first line of (53). The above values are of order $m^{2}+\mathcal{O}\left(m^{3} \sqrt{\xi}\right)$ for $\tilde{\tilde{\phi}}_{1}$ and $1 /(4 \xi)+\mathcal{O}(m / \sqrt{\xi})$ for the ghost $\left(\tilde{\tilde{\phi}}_{2}\right)$. Note that the correction to the mass of $\tilde{\tilde{\phi}}_{1}$ is suppressed only by $1 / M_{*}$ and is thus larger than the one discussed in the case of Kahler higher derivative terms, eq.(38) suppressed by $\xi=1 / M_{*}^{2}$. The effects of the operator $\Phi \square \Phi$ for phenomenology are discussed in Section 6 .

\subsection{The case of a general superpotential.}

The previous analysis is easily extended to an arbitrary superpotential in addition to the higher dimensional (derivative) term. Consider the Lagrangian

$$
\mathcal{L}=\int d^{4} \theta\left[\Phi^{\dagger} \Phi+S^{\dagger} S\right]+\left\{\int d^{2} \theta\left[s_{2} \sqrt{\xi} \Phi \square \Phi+W(\Phi, S)\right]+\text { h.c. }\right\}
$$

Here $W(\Phi, S)$ has no derivative terms in any of the fields, but otherwise is arbitrary 17 , and can include non-renormalisable interactions. $S$ is an arbitrary superfield. One shows that the Lagrangian equivalent to $\mathcal{L}$ is:

$$
\begin{aligned}
\mathcal{L}^{\prime} & =\int d^{4} \theta\left[\tilde{\Phi}_{1}^{\dagger} \tilde{\Phi}_{1}-\tilde{\Phi}_{2}^{\dagger} \tilde{\Phi}_{2}+S^{\dagger} S\right] \\
& +\left\{\int d^{2} \theta\left[\frac{1}{2}\left(d_{1} \tilde{\Phi}_{1}^{2}+d_{2} \tilde{\Phi}_{2}^{2}+2 d_{3} \tilde{\Phi}_{1} \tilde{\Phi}_{2}\right)+W\left(\Phi\left(\tilde{\Phi}_{1,2}\right), S\right)\right]+\text { h.c. }\right\}
\end{aligned}
$$

The coefficients $d_{1,2,3}$ are given in eqs.(60) and the relation between old and new fields is that of (61) which applies in this case too. Finally, $S$ is spectator under the unfolding of the fourth order theory into the second order one. The auxiliary fields are

$$
\begin{aligned}
-\tilde{F}_{1}^{*} & =d_{1} \tilde{\phi}_{1}+d_{3} \tilde{\phi}_{2}-\eta^{-1 / 4} W_{\phi}^{\prime} \\
\tilde{F}_{2}^{*} & =d_{2} \tilde{\phi}_{2}+d_{3} \tilde{\phi}_{1}+\eta^{-1 / 4} W_{\phi}^{\prime}, \quad-F_{s}^{*}=W_{\phi_{s}}^{\prime}
\end{aligned}
$$

\footnotetext{
${ }^{17}$ If higher derivative terms in $S$ exist in the superpotential, the same method is also applied for $S$.
} 
where $W_{\phi}^{\prime}\left(W_{\phi_{s}}^{\prime}\right)$ is the partial derivative wrt $\phi\left(\phi_{s}\right)$. Then the scalar potential is equal to $V=\left|\tilde{F}_{1}\right|^{2}-\left|\tilde{F}_{2}\right|^{2}+\left|\tilde{F}_{s}\right|^{2}$. Assume now that our model is in a ground state having (in the old basis) $F=F_{s}=0$ at the extremum point of the potential i.e. supersymmetry is unbroken. To picture this in the new formalism, use eq.(61) giving $\tilde{F}_{1}=\tilde{F}_{2}$. Further, the extremum conditions of the scalar potential wrt the new basis give three eqs which depend on $W_{\phi}^{\prime}$ and $W_{\phi_{s}}^{\prime}$ and on the second derivatives of the superpotential wrt $\phi, \phi_{s}$, evaluated at the extremum point considered. One also uses that $W_{\phi}^{\prime}=0$ and $W_{\phi_{s}}^{\prime}=0$ at the extremum point, while the second derivatives can be non-zero for this state. With these observations, one immediately finds that $\tilde{F}_{1}=\tilde{F}_{2}=0$ which recovers, in the new field basis that supersymmetry is unbroken, as expected by the equivalence of the two formulations.

The analysis can in principle be extended to the case when higher derivative terms of type discussed in sections 3.2, 4 are simultaneously present in the Kahler term and the superpotential, for an otherwise arbitrary superpotential. The method can also be applied to terms such as $\Phi^{n} \square \Phi$ in the superpotential or $\left(\Phi^{\dagger}\right)^{2} \Phi+$ h.c. etc, in the Kahler part of the action.

\section{$5 \quad$ Supersymmetry breaking and higher-derivatives}

\subsection{A model of supersymmetry breaking}

A natural question, which was our main motivation in studying theories with higher dimensional operators, is whether supersymmetry can be spontaneously broken due to the higher derivative terms, or equivalently in the two-derivative formulation, if supersymmetry breaking can be triggered by the presence of the ghost field(s).

The purpose of this section is to show the importance of the relation between the two formulations of a theory with higher derivatives found in the previous sections, for the case of supersymmetry breaking. For example one can have models with higher derivative terms which look rather uninteresting in the original (higher derivative) formulation and could be disregarded when decoupling the higher derivative term, and which in the two-derivative formulation are actually interacting theories and exhibit (spontaneous) supersymmetry breaking.

Here we provide an example of a model which in the limit of vanishing higher derivative operator has a trivial SUSY breaking, in the sense that the theory becomes free with a positive cosmological constant. However, in the presence of the higher derivative operator and in the second order formulation, the theory is interacting and has spontaneous supersymmetry 
breaking à la O'Raifeartaigh [42]. The example we consider starts from the two-derivative formulation with one particle (super)fields $S$ and two ghost superfields $\Phi, \chi$

$$
\mathcal{L}=\int d^{4} \theta\left(S^{\dagger} S-\Phi^{\dagger} \Phi-\chi^{\dagger} \chi\right)+\int d^{2} \theta\left[S\left(-\lambda \chi^{2}-m^{2}\right)-M_{*} \Phi \chi+\text { h.c. }\right] .
$$

The theory breaks SUSY à la O'Raifeartaigh since the SUSY conditions

$$
-F_{S}^{*}=-\lambda \phi_{\chi}^{2}-m^{2}=0 \quad, \quad-F_{\phi}^{*}=M_{*} \phi_{\chi}=0
$$

cannot be simultaneously satisfied. The vacuum of (69) is given by

$$
\left\langle\phi_{\chi}\right\rangle=\left\langle\phi_{\Phi}\right\rangle=0 \quad, \quad\left\langle\phi_{S}\right\rangle=v_{2}=\text { arbitrary }
$$

therefore the scale of SUSY breaking is given by $F_{S}^{*}=m^{2}$. In the limit of large ghost mass $M_{*} \gg m$, we can replace $\chi$ by the classical superfield eq.

$$
\chi=\frac{1}{4 M_{*}} \bar{D}^{2} \Phi^{\dagger} .
$$

Inserting (72) back into the original action (69) we find, after some standard manipulations, the Lagrangian 18

$$
\begin{aligned}
\mathcal{L} & =\int d^{4} \theta\left[S^{\dagger} S+\Phi^{\dagger} \Phi+\frac{1}{M_{*}^{2}} \Phi^{\dagger} \square \Phi+\frac{\lambda}{4 M_{*}^{2}}\left(S \Phi^{\dagger} \bar{D}^{2} \Phi^{\dagger}+\text { h.c. }\right)\right] \\
& +\left[\int d^{2} \theta\left(-m^{2} S\right)+\text { h.c. }\right] .
\end{aligned}
$$

Notice that it is safe to replace $\chi$ by (72) since in the original theory $\chi$ did not contribute to SUSY breaking. Notice also the sign flip in the kinetic term of $\Phi$, which became a standard kinetic term, supplemented by the two higher derivative operators. Of these operators the first one was considered already in Section 3, whereas the second one is of the form (d) of eq.(4), not considered before.

In the decoupling limit $M_{*} \rightarrow \infty$ eq.(73) describes a free supersymmetric theory for the two fields $S, \Phi$ with a linear superpotential $W=-m^{2} S$ which breaks supersymmetry in a trivial way, by adding a pure cosmological constant. Switching on the higher derivative terms generates an interacting theory whose SUSY breaking can be better described in the

\footnotetext{
${ }^{18}$ One can start in (69) with $+\Phi^{\dagger} \Phi$, then in (173) $\Phi^{\dagger} \square \Phi$ has opposite sign, see also (8), (18), (19).
} 
two-derivative version as an O'Raifeartaigh model (69). For $M_{*}^{2} \gg m^{2}$, both $\chi$ and $\Phi$ are in fact very heavy, whereas $S$ remains massless. We could have therefore integrated out $\Phi$ instead, in which case however we would turn $\chi$ into a particle-like superfield. It would be more satisfactory to integrate both $\chi, \Phi$ simultaneously. In this case, however, the theory for $S$ is non-local and highly non-linear.

Can we use the method developed in the previous sections to go from eq.(73) to (69)? The answer is indeed affirmative, despite the presence of the new term proportional to $\lambda$ in the Kahler term (not present before). This can be easily checked using eqs.(9), (46), (47), (48), for $\xi=1 / M_{*}^{2}$. The term in (73) proportional to $\lambda$ can be "moved" into the superpotential where it acquires an extra $\bar{D}^{2}$ and becomes of type $S\left(\bar{D}^{2} \Phi^{\dagger}\right)^{2}$ (using that $S$ is chiral), and which upon using second eq in (9) and (47) becomes a non-derivative interaction term. This interaction term recovers the first term in the superpotential in (69). One then uses that $\tilde{\mu}_{13}$ vanishes in the limit $\xi \rightarrow 0$ while $\tilde{\mu}_{23} \rightarrow-1 / \sqrt{\xi}$. The latter will in the end recover the last term in the superpotential of (69) (see also (46)). Finally, the first three D-terms in (73) become, using (9), the D-terms of (69) after disregarding the Kahler term of a non-interacting, massless superfield. This concludes our discussion on how to recover from (73), eq.(69).

It is important to notice that the formalism of previous sections applies not only in the presence of Gaussian-like terms (as it would be inferred from the discussion in Sections 314) but also for other terms, like the last D-term in (73). Finally, the method can be iterated for models with an even larger number of derivatives, to map it to a two-derivative theory. As a result the latter may then acquire higher dimensional superpotential interaction: 19 but no higher derivatives.

\subsection{Soft breaking terms}

We return to the models of Sections 314 of eqs.(8), (53) (or more generally eqs.(45), (66) 20 ), to comment on supersymmetry breaking. The results below apply to both of these models as we shall see shortly. Assuming that supersymmetry is broken by explicit soft terms [46] added to (8) and (53) respectively, let us investigate their explicit form in their second order, equivalent formulation. Consider therefore the addition of $\mathcal{L}_{\text {soft }}\left(\phi, \phi^{*}\right)$ to eq.(8), (53) where

$$
-\mathcal{L}_{\text {soft }}=M_{1}^{2} \phi^{*} \phi+\left(M_{2}^{2} \phi^{2}+h . c\right)
$$

\footnotetext{
${ }^{19}$ for a sufficiently large number of derivatives

${ }^{20}$ In this case soft terms in $S$ in addition to those below can also be present.
} 
Taking into account the relation between $\phi$ and $\tilde{\phi}_{i}$ which is similar for Sections 13 and 4 , see (47), (61), the soft terms become

$$
-\mathcal{L}_{\text {soft }}=\frac{M_{1}^{2}}{\sqrt{\beta}}\left|\tilde{\phi}_{1}-\tilde{\phi}_{2}\right|^{2}+\left[\frac{M_{2}^{2}}{\sqrt{\beta}}\left(\tilde{\phi}_{1}-\tilde{\phi}_{2}\right)^{2}+\text { c.c. }\right],
$$

where $\beta$ is equal to:

$$
\begin{array}{rlrl}
\eta & \equiv 1+4 \xi m^{2} & \text { (for Section 3.2) } \\
\eta^{\prime} \equiv 1+(17 / 4) \xi m^{2} & \text { (for Section 4) }
\end{array}
$$

which are thus of identical form up to a rescaling of $\xi$. Similar relations apply for trilinear soft terms. The soft breaking terms also acquired a scale/moduli dependence on $1 / \xi=M_{*}^{2}$ which is the scale of the higher derivative operators. This dependence is introduced dynamically by the "constraint" Lagrangian of eq.(11).

It is important to mention here that the presence of soft terms does not affect the holomorphic constraint and that the formalism we developed in previous sections is not affected. We checked this for specific cases by computing the spectrum after adding the soft terms, in both formulations (with 4- and 2-derivatives). In the second order formulation this was done using the eigenvalues of the second derivatives matrix of the potential with an appropriate metric in the field space, as detailed in previous sections.

\subsection{Further remarks on supersymmetry breaking}

We end this discussion with more general remarks on models with ghost superfields in the second order action. In these, the scalar potential is of the generic form

$$
V=\sum_{i}\left|F_{i}\right|^{2}-\sum_{j}\left|F_{j}\right|^{2}
$$

were the first sum accounts for contributions from particles and the second for that of the ghosts superfields present in the model considered. One could in principle have $V>0, V<0$ or even $V=0$ with broken supersymmetry. The breaking can in principle be done by vev's of the particle-like $F_{i} \neq 0$, by the ghost-like $F_{j} \neq 0$ 's or by both. For example a toy model with

$$
\mathcal{L}_{1}=\int d^{4} \theta\left[\Phi_{1}^{\dagger} \Phi_{1}-\Phi_{2}^{\dagger} \Phi_{2}\right]+\left[\int d^{2} \theta W\left(\Phi_{1}-\Phi_{2}\right)+\text { h.c. }\right]+m_{0}^{2}\left(\phi_{1}-\phi_{2}\right)^{2}
$$


can have a vanishing scalar potential, with broken supersymmetry. Indeed, the two auxiliary fields have identical eqs of motion, and thus $V\left(\phi_{1}, \phi_{2}\right)=V_{\text {soft }}\left(\phi_{1}, \phi_{2}\right)$ so $V$ has a minimum at $\phi_{1}-\phi_{2}=0$. Assuming $W^{\prime}\left(\phi_{1}-\phi_{2}\right) \neq 0$ which is easily satisfied if $W$ contains a linear term such as $g\left(\phi_{1}-\phi_{2}\right)$, then supersymmetry is broken, $F_{1}=F_{2}=g \neq 0$ yet the value of the scalar potential at the ground state is still vanishing. This remark has some similarities to [27] and may be of some interest for the cosmological constant problem [43].

At this point one could raise the issue of the stability [39, 41] of the models with higher derivative terms after supersymmetry breaking. Some stability issues were discussed in [41, where it was shown that the transition probabilities in such models have no exponential growth and in the decoupling limit $\left(M_{*} \rightarrow \infty\right)$ tend to those in ordinary second order theories. The price paid for stability is breaking unitarity at the scale $M_{*}$ which is assumed to be very high relative to all other scales in the theory.

In the supersymmetric context of our models, further analysis of the stability is necessary, along the lines discussed more recently in [39], where the possibility of the formation of a ghost condensate was analysed in similar models. Additional constraints 39] were also derived from imposing that the S-matrix respected all the standard axioms. Our purpose was to illustrate the method of "unfolding" the theory with higher dimensional operators (obtained for example after integrating out massive states) into a second order theory; this method is general and can be applied to models which eventually meet all the constraints discussed in [39]. We believe that our second-order formalism is very useful for a detailed analysis of stability, since it gives an off-shell description of the dynamics of the system, whereas in the original fourorder theory supersymmetry is realized on-shell, since auxiliary fields did acquire their own dynamics.

\subsection{Renormalisability issues.}

Using our formalism we showed that a theory with higher derivative operators of type considered in the previous sections, is equivalent to a theory without such operators but with additional superfields and renormalised couplings. Such equivalence remained true in the presence of soft breaking terms. If the initial theory had no other additional higher dimensional (non-derivative) operators, the equivalent second order formulation has only dimension 4 operators. Such theory can therefore be renormalisable. This is possible provided that we specify the analytical continuation of such theory to Euclidean metric. This is relevant since 
in models with ghosts the sign of the $i \epsilon$ prescription in their propagators is very important for the UV behaviour of a softly broken theory and in some cases can dramatically alter it, see discussion in 28] (despite a soft breaking and contrary to what one would expect on naive dimensional ground 21 ). However, if the propagators prescription for the ghost and particlelike degrees of freedom are similar (i.e they both undergo Wick rotations in same sense), then the Minkowski and Euclidean descriptions of the theory have similar UV behaviour. In this case, the 2-derivative formulation of the theory, which has only $\mathrm{D}=4$ operators and is softly broken, could actually be renormalisable 22 .

\section{Applications : MSSM with higher-derivative operators}

As an application to the possible low-energy effect of higher-derivative operators, we consider the Minimal Supersymmetric Standard Model (MSSM) and the corrections to the Higgs mass coming from such operators. We denote by $H_{1}, H_{2}$ the two MSSM Higgs doublets. The relevant Lagrangian to consider is that of the MSSM supplemented by derivative operators built out of the Higgs superfields. The lowest dimensional ones are23:

$$
\mathcal{L}=\mathcal{L}_{\mathrm{MSSM}}+\int d^{4} \theta\left[\xi_{1} H_{1}^{\dagger} \square H_{1}+\xi_{2} H_{2}^{\dagger} \square H_{2}\right]-\left[\int d^{2} \theta \sqrt{\xi_{3}} H_{1} \square H_{2}+\text { h.c. }\right],
$$

where $\mathcal{L}_{\text {MSSM }}$ is the standard MSSM Lagrangian, including the soft-breaking terms. Notice first of all that the higher derivative terms do not change the vacuum structure of the theory. They change however the tree-level Higgs physical spectrum. Indeed, by expanding the Lagrangian (79) around the vacuum breaking the electroweak symmetry and restricting for simplicity to the case $\xi_{3}=0$, we find the Lagrangian relevant for the scalar sector 24

$$
\mathcal{L}^{(2)}=-h_{i}^{\dagger}\left[\xi_{i} \square^{2}+\square\right] h_{i}+\xi_{i} F_{i}^{*} \square F_{i}-V, \quad i=1,2
$$

where $V$ is that of the MSSM before eliminating the auxiliary $F_{1,2}$ of $H_{1,2}$. One computes the corrected values of $m_{h}^{2}, m_{H}^{2}$ of the neutral scalar eigenstates, using the poles of the

\footnotetext{
${ }^{21}$ In the presence of higher derivative terms power counting for UV divergence of loop integrals does not always work in Minkowski space, for details see [28].

${ }^{22}$ It would be useful to derive such prescriptions from the original theory with higher derivative operators using a path integral formulation in the Minkowski space-time. No such formulation is available at present.

${ }^{23}$ The effects of gauge interactions are not included in this section.

${ }^{24}$ Here $h_{i}$ are the scalar Higgs components of the superfields.
} 
corresponding propagators (vanishing of the appropriate determinant in the basis of Higgs and auxiliary fields). Assuming for simplicity that $\xi_{1}=\xi_{2}=\xi$, then one finds

$$
2 \xi p^{4}-p^{2}\left[1+\left(m_{h}^{2}-\mu^{2}\right) \xi\right]+m_{h}^{2}=0 \quad, \quad 2 \xi p^{4}-p^{2}\left[1+\left(m_{H}^{2}-\mu^{2}\right) \xi\right]+m_{H}^{2}=0,
$$

where $m_{h}$ and $m_{H}$ are the masses computed in MSSM. Setting $p^{2}$ equal to the corrected corresponding Higgs mass, we find the leading corrections to the neutral Higgs masses to be

$$
\frac{\delta m_{h}^{2}}{m_{h}^{2}} \approx \xi\left(m_{h}^{2}+\mu^{2}\right) \quad, \quad \frac{\delta m_{H}^{2}}{m_{H}^{2}} \approx \xi\left(m_{H}^{2}+\mu^{2}\right) .
$$

For a cutoff $M_{*}\left(\xi_{1,2}=1 / M_{*}^{2}\right)$ in the $5-10 \mathrm{TeV}$ range, these effects are of order $1-2 \%$ and therefore too small to give a sizable contribution.

Let us now examine the effects of the operator $W_{1}=\sqrt{\xi}_{3} H_{1} \square H_{2}$ in the superpotential, where $\sqrt{\xi}_{3}=1 / M_{*}^{2}$ and set $\xi_{1}=\xi_{2}=0$. It turns out that these can be substantial, since $W_{1}$ is of dimension 4 and therefore of the same order as the non-derivative operator $W^{\prime}=$ $\left(H_{1} H_{2}\right)^{2} / M_{*}$ considered in [48, 49]. To investigate these effects, first notice that despite the presence of the derivative in the superpotential, the auxiliary fields of $H_{1}, H_{2}$ are not dynamical and can be eliminated by their eqs of motion. After doing so, one finds a Lagrangian for the scalar components

$$
\mathcal{L}=-h_{i}^{\dagger}\left(\square+\xi_{3} \square^{2}-2 \mu \sqrt{\xi_{3}} \square\right) h_{i}-V, \quad i=1,2
$$

where $V$ is that of the MSSM. Finding the extrema of the potential, going to the mass eigenstates $(h, H)$ etc, proceeds as in the MSSM, while the kinetic terms are invariant under these transformations. One then finds the poles of the propagators above from

$$
-p^{2}+\xi_{3} p^{4}+2 \mu \sqrt{\xi_{3}} p^{2}+m_{h}^{2}=0
$$

where $m_{h}$ is the value computed in the MSSM. With $p^{2}=m_{h}^{2}+\delta m_{h}^{2}$, the effect of $W_{1}$ on the lightest Higgs mass is found to be

$$
\frac{\delta m_{h}^{2}}{m_{h}^{2}} \approx 2 \mu \sqrt{\xi}_{3}=\frac{2 \mu}{M_{*}} .
$$

This correction is of order $10 \%$ for $M_{*} \sim 10 \mathrm{TeV}$ and $\mu \sim 500 \mathrm{GeV}$ and can therefore increase the Higgs mass above the experimental limit even before adding the quantum corrections ! Such a correction is comparable to the one found in [48, 49] using the operator $\left(H_{1} H_{2}\right)^{2} / M_{*}$. For a further discussion of these corrections see also [44]. 


\section{Conclusions}

Higher dimensional operators (derivative or otherwise) are a common presence in $4 \mathrm{D}$ effective, nonrenormalisable theories. They are easily generated in the low-energy effective action from $4 \mathrm{D}$ renormalisable theories upon integration out massive (super)fields. Such operators are also dynamically generated by radiative corrections in effective theories of compactification even for the simplest orbifolds.

Motivated by this, we investigated in detail the case of $4 \mathrm{D} N=1$ supersymmetric models with such operators. Using a superfield language it was shown that a $4 \mathrm{D} N=1$ supersymmetric theory with higher derivative terms in the Kahler potential and an arbitrary superpotential is equivalent to a $4 \mathrm{D} \mathrm{N}=1$ theory of second order with two additional superfields and renormalised interactions. Because in the initial, higher derivative formulation of the theory both $\square \phi$ and the auxiliary field $F$ where propagating, by supersymmetry this lead in the two-derivative formulation of the theory to the existence of the two additional superfields mentioned above.

The method developed was then extended to the case of $4 \mathrm{D} \mathrm{N}=1$ models with higher derivative terms in the superpotential whose remaining part is otherwise arbitrary. It was again showed that such model is equivalent to a $4 \mathrm{D} \mathrm{N}=1$ second order theory with an additional (ghost) superfield and renormalised couplings. Unlike the case of higher derivatives in the Kahler potential, in this case there is only one additional superfield in the second-order formulation because in this case only $\square \phi$ is propagating in the higher derivative theory, and this implied, by supersymmetry, the existence of one additional (ghost) superfield (indeed, we found that $\tilde{\Phi}_{3}$ was acting only as a constraint superfield in Section 4 , whereas it was a propagating degree of freedom in Section 3 ). Finally, it was verified in both cases that in the second order formulations of the theory the spectrum must be computed with an appropriate metric in field space to account for the negative kinetic terms of the ghosts fields.

In both cases the couplings of the new, second order theory, acquire already at the tree level a dependence on the scale of the higher dimensional operator. The new, second order formulation of the theory has the advantage of providing a standard, familiar framework for investigating the role of these operators in explicit models. We argued that if there are no additional operators of $D>4$ in the original theory apart from the higher derivative ones considered, the second order formulation of the theory has only $\mathrm{D}=4$ operators. This theory can be renormalisable, under some assumptions for the analytical continuation from 
the Minkowski to Euclidean metric. This requires that the ghost propagators be Wick rotated to Euclidean space in the same sense as the particle-like ones, leading to similar UV for both Minkowski and Euclidean descriptions.

In the new basis of the second order theory, the original superfield is a mixing of particle and ghost-like superfields, and thus the particle-like degrees of freedom are not identical in the two descriptions. This brings an intriguing issue, regarding which of the two descriptions is more fundamental. Ultimately this refers to which choice of the degrees of freedom one should make for the particle-like field: original field of the fourth order theory or the particle-like degree of freedom in the second order theory. This issue is relevant particularly at the loop level, when superfields re-scaling anomalies associated with the transformations we performed, can bring in quantum corrections to the equivalence of the two formulations.

Our analysis remains valid in the presence of supersymmetry breaking terms, as it was confirmed by computing the spectrum in both formulations of the theory with higher derivative operators, for explicit forms of soft breaking terms. The higher derivative operators are also important for supersymmetry breaking. We showed that models with higher derivative terms which look rather un-interesting in the original formulation and could be disregarded when decoupling these terms, turn out to be in the two-derivative formulation, interacting theories that exhibit spontaneous supersymmetry breaking à la O'Raifeartaigh.

The analysis can be applied in the presence of arbitrary higher derivative terms, using eventually an iteration of the method presented in Sections 3 and 4 . Higher dimensional Kahler terms other that those leading to standard kinetic terms in the two-derivative formulation, can be "moved" into the superpotential with an additional (super)derivative and become, in the new field basis, higher dimensional non-derivative interactions. An example of this type was discussed in the second part of Section 5.1. Similar techniques can be applied in the case of complicated derivative interactions in the superpotential, by replacing derivatives of superfields with new superfields and appropriate holomorphic constraints in the Lagrangian. Finally, in specific cases and for appropriate parameters in the original theory, some of the Kahler terms of ghost superfields that can emerge in the two-derivative formulation may in some cases decouple from the Lagrangian, if these fields do not have superpotential terms. To conclude, our method shows that theories with higher derivative operators can be mapped to theories with higher dimensional, non-derivative operators.

An application to the case of higher derivative terms in the MSSM Lagrangian was also 
presented. It was estimated that the Higgs mass can be lifted above the experimental limit by such terms, even before adding quantum corrections associated with them. Regardless of the exact nature of the ghost fields that higher derivative operators bring in (i.e. whether these fields exist as asymptotic states or only loop ones), the method we presented can be used as a perturbative tool to investigate the effects on low energy physics of new high-scale physics associated with higher derivative operators, much in the same way this is done for higher dimensional operators.

In general the presence of ghost superfields in a $4 \mathrm{D} \mathrm{N}=1$ action has as effect that the scalar potential of such theory is not positive definite. Therefore, the vanishing of $V$ is not equivalent to exact supersymmetry anymore and one can have $V>0, V<0$ and even $V=0$ for broken supersymmetry. In the last case $\left|\tilde{F}_{i}\right|=\left|\tilde{F}_{j}\right| \neq 0$ where $i$ and $j$ label the contributions of particle and ghost-like states to $V$. A vanishing scalar potential would require the breaking of supersymmetry be done by both the ghost and particle-like degrees of freedom. This last case could be of some interest for the cosmological constant problem.

We would like to end our discussion of the equivalence on the two formulations of the theory with higher dimensional operators with the following observation. The equivalence we showed between the fourth order and second order formulation is valid at the classical level. A legitimate question is then whether one can make similar claims of equivalence at the quantum level. Although the question is beyond the purpose of this paper, let us make the following remark. The study of the quantum equivalence is somewhat beyond the possibility of an effective field theory framework, where the absence of a detailed UV completion would render such analysis incomplete or valid in very specific cases only. Nevertheless, restricting ourselves to the lagrangian with one higher derivative operator, we performed an explicit check of the equivalence at the one-loop level, for the radiative correction to the mass of the original scalar field $\phi$, after a soft breaking of supersymmetry. Using (8) and its second order formulation (46) with (47), (48), we checked explicitly that one obtains the same one-loop result. This is interesting in itself and checks the validity of our formalism at the quantum level too, for this particular case. However, given the effective character of these theories and the absence of a UV completion, one should be careful not to extrapolate this finding to a general, similar statement of quantum equivalence of the two formulations. 


\section{Acknowledgements}

This work was partially supported by INTAS grant, 03-51-6346, RTN contracts MRTN-CT2004-005104 and MRTN-CT-2004-503369, CNRS PICS \# 2530, 3059 and 3747, and by a European Union Excellence Grant, MEXT-CT-2003-509661. D.G. also acknowledges the partial support of the Marie Curie Research Training Network of the European Community, contract $n$. MRTN-CT-2006-035863 and that of a CERN visiting fellowship.

\section{Appendix}

In the case of higher derivatives of Section 3 the eigenvalues were

$$
\nu_{1}=\frac{1}{2}(1+\sqrt{\eta}), \quad \nu_{2}=\frac{1}{2}(1-\sqrt{\eta}), \quad \nu_{3}=-\frac{\xi m^{2}}{16}, \quad\left(\eta \equiv 1+4 \xi m^{2}\right)
$$

The corresponding eigenvectors $v_{i j}$ with $\Phi_{i}^{\prime}=v_{i j} \Phi_{j}$ (see Section 3.2) are respectively

$$
\begin{aligned}
& v_{1 j}=\frac{1}{\left\|v_{1}\right\|}\left\{-e^{i h_{1}} \frac{\nu_{1}}{m \sqrt{\xi}} \cos \theta,-e^{-i\left(h-h_{1}\right)} \frac{\nu_{1}}{m \sqrt{\xi}} \sin \theta, 1\right\}_{j} \\
& v_{2 j}=\frac{1}{\left\|v_{2}\right\|}\left\{-e^{i h_{1}} \frac{\nu_{2}}{m \sqrt{\xi}} \cos \theta,-e^{-i\left(h-h_{1}\right)} \frac{\nu_{2}}{m \sqrt{\xi}} \sin \theta, 1\right\}_{j} \\
& v_{3 j}=\frac{1}{\left\|v_{3}\right\|}\left\{-e^{i h} \tan \theta, 1,0\right\}_{j}, \quad j=1,2,3 .
\end{aligned}
$$

with $\left\|v_{i}\right\|, i=1,2,3$, the norm of the corresponding vector.

In the case of higher derivatives of Section 4 the eigenvalues were

$$
\nu_{1}=\frac{1}{2}\left(1+\sqrt{\eta^{\prime}}\right), \quad \nu_{2}=\frac{1}{2}\left(1-\sqrt{\eta^{\prime}}\right), \quad \nu_{3}=0, \quad \eta^{\prime} \equiv 1+4 \xi m^{2}\left(1+s_{2}^{2} / 16\right)
$$

The corresponding eigenvectors used there were

$$
\begin{aligned}
& v_{1 j}=\frac{1}{\left\|v_{1}\right\|}\left\{\frac{-\nu_{1} e^{i h_{1}}}{m \sqrt{\xi}} \cos \theta+\frac{e^{-i\left(h_{1}-h\right)} s_{2}}{4} \sin \theta, \frac{-s_{2} e^{-i h_{1}}}{4} \cos \theta-\frac{\nu_{1} e^{-i\left(h-h_{1}\right)}}{m \sqrt{\xi}} \sin \theta, 1\right\}_{j} \\
& v_{2 j}=\frac{1}{\left\|v_{2}\right\|}\left\{\frac{-\nu_{2} e^{i h_{1}}}{m \sqrt{\xi}} \cos \theta+\frac{s_{2} e^{-i\left(h_{1}-h\right)}}{4} \sin \theta, \frac{-s_{2} e^{-i h_{1}}}{4} \cos \theta-\frac{\nu_{2} e^{-i\left(h-h_{1}\right)}}{m \sqrt{\xi}} \sin \theta, 1\right\}_{j} \\
& v_{3 j}=\frac{1}{\left\|v_{3}\right\|}\left\{\frac{-4}{s_{2}} e^{i\left(h-h_{1}\right)} \sin \theta, \frac{4}{s_{2}} e^{-i h_{1}} \cos \theta, 1\right\}_{j}
\end{aligned}
$$




\section{References}

[1] S. Groot Nibbelink and M. Hillenbach, "Renormalization of supersymmetric gauge theories on orbifolds: Brane gauge couplings and higher derivative operators," Phys. Lett. B 616 (2005) 125 arXiv:hep-th/0503153; S. Groot Nibbelink and M. Hillenbach, "Quantum Corrections to Non-Abelian SUSY Theories on Orbifolds," E-print: arXiv:hep-th/0602155.

[2] D. M. Ghilencea, "Higher derivative operators as loop counterterms in one-dimensional field theory orbifolds" JHEP 0503 (2005) 009 arXiv:hep-ph/0409214 D. M. Ghilencea and H. M. Lee, "Higher derivative operators from transmission of supersymmetry breaking on S(1)/Z(2)," JHEP 0509 (2005) 024 hep-ph/0505187]; "Higher derivative operators from Scherk-Schwarz supersymmetry breaking on $T^{* * 2 / Z(2), " ~ J H E P ~} 0512$ (2005) 039 arXiv:hep-ph/0508221];

[3] D. M. Ghilencea, Hyun Min Lee, K. Schmidt-Hoberg "Higher Derivatives and branelocalised kinetic terms in gauge theories on orbifolds.", E-print: arXiv:hep-ph/0604215. D. M. Ghilencea, "Compact dimensions and their radiative mixing," Phys. Rev. D 70 (2004) 045018 arXiv:hep-ph/0311264.

[4] J. F. Oliver, J. Papavassiliou and A. Santamaria, "Can power corrections be reliably computed in models with extra dimensions?," Phys. Rev. D 67 (2003) 125004 arXiv:hep-ph/0302083.

[5] E. Alvarez, A. F. Faedo, "Renormalized Kaluza-Klein theories," arXiv:hep-th/0602150.

[6] A. Lewandowski and R. Sundrum, "RS1, Higher Derivatives and Stability" Phys. Rev. D 65 (2002) 044003 arXiv:hep-th/0108025].

[7] C. Armendariz-Picon, T. Damour and V. F. Mukhanov, "k-inflation," Phys. Lett. B 458 (1999) 209 arXiv:hep-th/9904075]; J. Garriga and V. F. Mukhanov, "Perturbations in k-inflation," Phys. Lett. B 458 (1999) 219 arXiv:hep-th/9904176]; C. ArmendarizPicon, V. F. Mukhanov and P. J. Steinhardt, "Essentials of k-essence," Phys. Rev. D 63 (2001) 103510 arXiv:astro-ph/0006373.

[8] A. Anisimov, E. Babichev and A. Vikman, "B-inflation," JCAP 0506 (2005) 006 arXiv:astro-ph/0504560]; M. Z. Li, B. Feng and X. M. Zhang, "A single scalar field 
model of dark energy with equation of state crossing -1," JCAP 0512 (2005) 002 arXiv:hep-ph/0503268.

[9] G. W. Gibbons, "Phantom matter and the cosmological constant," E-print: arXiv:hep-th/0302199.

[10] V. Branchina, H. Mohrbach, J. Polonyi, "The antiferromagnetic Phi**4 model. I: The mean-field solution," Phys. Rev. D 60 (1999) 045006 [hep-th/9612110]; "The antiferromagnetic phi**4 model. II: The one-loop renormalization,” Phys. Rev. D 60 (1999) 045007 hep-th/9612111.

[11] A.A. Slavnov, "Renormalisable electroweak model without fundamental scalar mesons", E-print: arXiv:hep-th/0601125; "Higgs mechanism as a collective effect due to extra dimension," Theor. Math. Phys. 148 (2006) 1159 [Teor. Mat. Fiz. 148 (2006) 339] arXiv:hep-th/0604052.

[12] K. Jansen, J. Kuti and C. Liu, "The Higgs model with a complex ghost pair," Phys. Lett. B 309 (1993) 119 arXiv:hep-lat/9305003.

[13] G. F. Giudice, C. Grojean, A. Pomarol and R. Rattazzi, "The Strongly-Interacting Light Higgs," E-print: arXiv:hep-ph/0703164.

[14] K.S. Stelle, "Renormalisation of Higher Derivative Quantum Gravity" Phys. Rev. D 16 (1977) 953.

[15] R. C. Myers, "Higher Derivative Gravity, Surface terms and String Theory," Phys. Rev. D 36 (1987) 392.

[16] S. Ferrara, B. Zumino, "Structure of linearised supergravity and conformal supergravity", Nucl. Phys. B134 (1978), 301.

[17] N.V.Krasnikov, A.B. Kyiatkin and E.R.Poppitz, "Structure of the effective potential in supersymmetric theories with higher order derivatives coupled to supergravity" Phys. Lett. B 222, (1989) 66.

[18] I. Antoniadis, E.T. Tomboulis, "Gauge invariance and Unitarity in Higher Derivative Quantum Gravity", Phys. Rev. D33, (1986) 2756. 
[19] E. T. Tomboulis, "Unitarity In Higher Derivative Quantum Gravity," Phys. Rev. Lett. 52 (1984) 1173.

[20] H. W. Hamber and R. M. Williams, "Higher Derivative Quantum Gravity On A Simplicial Lattice," Nucl. Phys. B 248 (1984) 392 [Erratum-ibid. B 260 (1985) 747].

[21] S. Nojiri and S. D. Odintsov, "Brane-world cosmology in higher derivative gravity or warped compactification in the next-to-leading order of AdS/CFT correspondence," JHEP 0007 (2000) 049 arXiv:hep-th/0006232.

[22] S. L. Dubovsky, "Phases of massive gravity," JHEP $\mathbf{0 4 1 0}$ (2004) 076 arXiv:hep-th/0409124].

[23] S. Cecotti, S. Ferrara and L. Girardello, "Flat Potentials In Higher Derivative Supergravity," Phys. Lett. B 187 (1987) 327. "Structure of the scalar potential in general N=1 Higher derivative supergravity in four dimensions," Phys. Lett. B 187 (1987) 321; S. Cecotti, S. Ferrara, L. Girardello, M. Porrati and A. Pasquinucci, "Matter Coupling In Higher Derivative Supergravity," Phys. Rev. D 33 (1986) 2504; S. Cecotti, S. Ferrara, M. Porrati and S. Sabharwal, "New Minimal Higher Derivative supergravity coupled to matter," Nucl. Phys. B 306 (1988) 160.

[24] I. G. Avramidi and A. O. Barvinsky, "Asymptotic Freedom In Higher Derivative Quantum Gravity," Phys. Lett. B 159 (1985) 269.

[25] D. A. Eliezer and R. P. Woodard, "The Problem Of Nonlocality In String Theory," Nucl. Phys. B 325 (1989) 389.

[26] A. M. Polyakov, "Fine Structure of Strings," Nucl. Phys. B 268 (1986) 406.

[27] D. E. Kaplan and R. Sundrum, "A symmetry for the cosmological constant," arXiv:hep-th/0505265.

[28] I. Antoniadis, E. Dudas and D. M. Ghilencea, "Living with ghosts and their radiative corrections," Nucl.Phys. B767 (2007) 29; arXiv:hep-th/0608094.

[29] V. V. Nesterenko, "On the instability of classical dynamics in theories with higher derivatives," Phys. Rev. D 75 (2007) 087703 arXiv:hep-th/0612265. 
[30] B. Grinstein, D. O'Connell and M. B. Wise, "The Lee-Wick standard model," arXiv:0704.1845 [hep-ph]

[31] J. R. Espinosa, B. Grinstein, D. O'Connell and M. B. Wise, "Neutrino masses in the Lee-Wick standard model," arXiv:0705.1188 [hep-ph].

[32] A. Hebecker and J. March-Russell, "Proton decay signatures of orbifold GUTs," Phys. Lett. B 539 (2002) 119 arXiv:hep-ph/0204037.

[33] W. Buchmuller, L. Covi, D. Emmanuel-Costa and S. Wiesenfeldt, "Flavour structure and proton decay in 6D orbifold GUTs," JHEP 0409 (2004) 004 arXiv:hep-ph/0407070].

[34] A. A. Slavnov, "The Pauli-Villars Regularization For Non-Abelian Gauge Theories. (In Russian)," Teor. Mat. Fiz. 33 (1977) 210. "Invariant regularization of gauge theories," Teor. Mat. Fiz. 13 (1972) 174.

[35] C. P. Martin and F. Ruiz Ruiz, "Higher Covariant Derivative Pauli-Villars Regularization Does Not Lead To A Consistent QCD," Nucl. Phys. B 436 (1995) 545 arXiv:hep-th/9410223.

[36] M. Asorey and F. Falceto, "On the consistency of the regularization of gauge theories by high covariant derivatives," Phys. Rev. D 54 (1996) 5290 arXiv:hep-th/9502025].

[37] T. D. Bakeyev and A. A. Slavnov, "Higher covariant derivative regularization revisited," Mod. Phys. Lett. A 11 (1996) 1539 arXiv:hep-th/9601092.

[38] A. V. Smilga, "Ghost-free higher-derivative theory," Phys. Lett. B 632 (2006) 433 arXiv:hep-th/0503213]. "Benign vs. malicious ghosts in higher-derivative theories," Nucl. Phys. B 706 (2005) 598 hep-th/0407231]. D. Robert and A. V. Smilga, "Supersymmetry vs ghosts," arXiv:math-ph/0611023.

[39] N. Arkani-Hamed, H. C. Cheng, M. A. Luty and S. Mukohyama, "Ghost condensation and a consistent infrared modification of gravity," JHEP 0405 (2004) 074 arXiv:hep-th/0312099]. A. Adams, N. Arkani-Hamed, S. Dubovsky, A. Nicolis and R. Rattazzi, "Causality, analyticity and an IR obstruction to UV completion," JHEP 0610 (2006) 014 arXiv:hep-th/0602178. 
[40] J. H. Leon, C. P. Martin and F. Ruiz Ruiz, "Unitarity violation in non-Abelian PauliVillars regularization," Phys. Lett. B 355 (1995) 531 arXiv:hep-ph/9507443]; K. Fujikawa, "Generalized Pauli-Villars regularization and the covariant form of anomalies," Nucl. Phys. B 428 (1994) 169 arXiv:hep-th/9405166]; E. Elizalde, "On the relation between the generalized Pauli-Villars and the covariant regularization," Phys. Lett. B 342 (1995) 277.

[41] S. W. Hawking, T. Hertog, "Living with ghosts", Phys. Rev. D 65 (2002) 103515 hep-th/0107088]. S. W. Hawking, in "Quantum Field theory and Quantum Statistics: Essays in Honour of the 60 th Birthday of E.S. Fradkin", eds. A.Batalin, C.J.Isham, C.A. Vilkovisky, Hilger, Bristol, UK (1987).

[42] L. O'Raifeartaigh, "Spontaneous Symmetry Breaking For Chiral Scalar Superfields," Nucl. Phys. B 96 (1975) 331.

[43] S. Weinberg, "The cosmological constant problem," Rev. Mod. Phys. 61 (1989) 1.

[44] I. Antoniadis, E. Dudas, D. M. Ghilencea, P. Tziveloglou, work in progress.

[45] W. Siegel, "Hidden Ghosts," Phys. Lett. B 93 (1980) 170.

[46] L. Girardello, M.T. Grisaru, "Soft breaking of Supersymmetry", Nucl. Phys. B 194 (1982) 65

[47] D. I. Kazakov and G. S. Vartanov, "Renormalizable $1 / N_{f}$ Expansion for Field Theories in Extra Dimensions," arXiv:0707.2564 [hep-th]; "Renormalizable expansion for nonrenormalizable theories. II: Gauge higher dimensional theories," arXiv:hep-th/0702004; "Renormalizable expansion for nonrenormalizable theories. I: Scalar higher dimensional theories," arXiv:hep-th/0607177.

[48] A. Strumia, "Bounds on Kaluza-Klein excitations of the SM vector bosons from electroweak tests," Phys. Lett. B 466 (1999) 107 arXiv:hep-ph/9906266]; A. Brignole, J. A. Casas, J. R. Espinosa and I. Navarro, "Low-scale supersymmetry breaking: Effective description, electroweak breaking and phenomenology," Nucl. Phys. B 666 (2003) 105 arXiv:hep-ph/0301121. 
[49] N. Seiberg, Talk at "Strings 2007", 25-29 June 2007, Madrid (Spain); M. Dine, N. Seiberg and S. Thomas, "Higgs Physics as a Window Beyond the MSSM (BMSSM)," arXiv:0707.0005 [hep-ph]. 